Алгебра и анализ

Том. 16 (2004), вып. 1
St. Petersburg Math. J. Vol. 16 (2005), No. 1, Pages 105-142 S 1061-0022(04)00845-3

Article electronically published on December 14, 2004

\title{
ON THE RIEMANN-HILBERT-BIRKHOFF INVERSE MONODROMY PROBLEM AND THE PAINLEVÉ EQUATIONS
}

\author{
A. A. BOLIBRUCH, A. R. ITS, AND A. A. KAPAEV
}

This paper is dedicated to Professor M. Sh. Birman on the occasion of his 75th birthday

\begin{abstract}
A generic $2 \times 2$ system of first order linear ordinary differential equations with second degree polynomial coefficients is considered. The problem of finding such a system with the property that its Stokes multipliers coincide with a given set of relevant $2 \times 2$-matrices constitutes the first nontrivial case of the RiemannHilbert-Birkhoff inverse monodromy problem. The meromorphic (with respect to the deformation parameter) solvability of this problem is proved. The approach is based on Malgrange's generalization of the classical Birkhoff-Grothendieck theorem to the case with the parameter. As a corollary, a new proof of meromorphicity of the second Painlevé transcendent is obtained. An elementary proof of a particular case of Malgrange's theorem, needed for our goals, is also presented (following an earlier work of the first author).
\end{abstract}

\section{§1. INTRODUCTION}

In this paper we study the inverse monodromy problem, which we shall also refer to as the Riemann-Hilbert-Birkhoff problem or simply as the Riemann-Hilbert problem, for a $2 \times 2$ linear system of ordinary differential equations with rational coefficients. The system is assumed to have only one irregular singular point of Poincaré index 3. We shall consider a generic situation, which means that, up to trivial gauge and scaling transformations, the system in question can be written in the following form:

$$
\begin{aligned}
\frac{d \Psi}{d \lambda} & =A(\lambda) \Psi(\lambda), \\
A(\lambda) & =A_{2} \lambda^{2}+A_{1} \lambda+A_{0} \\
& =-4 i \lambda^{2} \sigma_{3}+4 i \lambda\left(\begin{array}{cc}
0 & u \\
-v & 0
\end{array}\right)+\left(\begin{array}{cc}
-i x-2 i u v & -2 w \\
-2 y & i x+2 i u v
\end{array}\right), \\
\sigma_{3} & =\left(\begin{array}{cc}
1 & 0 \\
0 & -1
\end{array}\right),
\end{aligned}
$$

2000 Mathematics Subject Classification. Primary 34M55, 34M50, 34M40.

Key words and phrases. Riemann-Hilbert problem, Painlevé equations.

The first author was supported in part by RFBR (grant no. 99-01-00157) and by INTAS (grant no. 97-1644). The second author was supported in part by NSF (grants nos. DMS-9801608 and DMS0099812). The third author was supported in part by RFBR (grant no. 99-01-00687). The second author also thanks the Département de Mathématiques, Université de Strasbourg, where part of this work was done, for hospitality during his visit. 
where the complex numbers $u, v, w, y$, and $x$ form a parameter set for the class of linear systems considered. We shall denote this set by

$$
\mathcal{A} \equiv\{(u, v, w, y, x)\}
$$

and, as in [24], we shall call it the set of singular data of system (11). Note that the set $\mathcal{A}$ can be thought of as a complex manifold isomorphic to $\mathbb{C}^{5}$, and hence

$$
\operatorname{dim}_{\mathbb{C}} \mathcal{A}=5 .
$$

The Riemann-Hilbert-Birkhoff problem is posed as the question on surjectivity for the generalized monodromy map,

$$
\mathcal{A} \mapsto \mathcal{M},
$$

where $\mathcal{M}$ stands for the set of generalized monodromy data. The set $\mathcal{M}$ is responsible for the global properties of the solutions of a linear system. In the case of system (1D), the set of generalized monodromy data consists of (a) the two parameters of the exponential part of the formal solution at $\lambda=\infty$, which is the only singular point of the system, and (b) the six Stokes matrices associated with this (irregular) singular point. The collection $\mathcal{S}$ of the Stokes matrices forms the set of monodromy data for system (1). The monodromy map,

$$
\mathcal{A} \mapsto \mathcal{S},
$$

is the transcendental part of the generalized monodromy map (44). The detailed definition of the sets $\mathcal{S}$ and $\mathcal{M}$ will be given in the next section. In the case of a general linear system, the set of monodromy data includes also the corresponding monodromy group (which is trivial for system (10) and the connection matrices (matrices that connect the marked fundamental solution with the local fundamental solutions at different singular points).

In a more general sense, the Riemann-Hilbert problem includes the study of the analytic properties of the inverse generalized monodromy map. A question of particular interest is as to whether the inverse generalized monodromy map is a meromorphic function on the submanifold $\mathcal{D} \equiv \mathcal{M} \backslash \mathcal{S}$.

Our interest in the inverse monodromy problem for system (1) is twofold. The first reason is that this is the first nontrivial case of the Riemann-Hilbert-Birkhoff problem for systems with irregular singular points. Indeed, the two preceding cases, i.e., $2 \times 2$ systems with the coefficient matrices $A(\lambda)=A_{1} \lambda+A_{0}$ and $A(\lambda)=A_{0}$, can be solved explicitly in terms of contour integrals of elementary functions (i.e., in terms of the confluent hypergeometric function) and simply in terms of elementary functions, respectively. Hence, for the two preceding cases, the monodromy maps (4) and (5) can be analyzed by using explicit formulas that involve at most the contour integrals.

The second reason is the relationship between (11) and the integrable systems, in particular, the theory of Painlevé equations. This relationship occurs due to the fact that, simultaneously, system (1) is the first case of a polynomial system that allows for nontrivial isomonodromy deformations, i.e., the monodromy map (5) has nontrivial level curves. Indeed, in the case of the linear matrix-valued function $A(\lambda)$, there are no free parameters (after the proper gauge and scaling transformations) in the exponential part of the formal solutions, the sets $\mathcal{S}$ and $\mathcal{M}$ coincide, and the monodromy map (5) is one-to-one, i.e., no "room" remains for isomonodromy deformations.

The isomonodromy deformations of the linear system (1) are transformed into a certain nonlinear dynamical system on the manifold $\mathcal{A}$. In turn, this nonlinear system can be reduced to the Painlevé-2 or Painlevé-34 equations (more details are given in $\S 3$ and in Appendix 2). A similar isomonodromy interpretation, with different rational matrices $A(\lambda)$, exists for every Painlevé equation, and this fact was discovered at the turn of the 
last century by R. Garnier and L. Schlesinger. What apparently was not noticed in the classical works, is that the associated Riemann-Hilbert problems can be used to study the global properties of the Painlevé functions and, in particular, to obtain the explicit asymptotic connection formulas. The latter is a modern achievement, which is based on the isomonodromy method in the theory of Painlevé equations, initiated in the papers [24] and [14]. We refer the reader to the monograph [19] and to the survey papers [15, 18, 21] for more details on the isomonodromy method and the connection formulas for Painlevé equations.

The questions about surjectivity of the monodromy map and the property of the inverse generalized monodromy map to be meromorphic play an important role in the theory of Painlevé equations. The positive resolution of the first question justifies the very basic technical idea of the isomonodromy method, which is the parametrization of the solutions of Painlevé equations by the points of the relevant manifolds of monodromy data. To have a rigorous proof of the existence of such a parametrization is crucial for rigorous global asymptotic analysis of Painlevé functions (see, e.g., [21, 22]). An immediate consequence of a positive answer to the second question is the principal property of Painlevé equations, called the Painlevé property, saying that all their solutions are meromorphic on the universal covering of a punctured Riemann sphere determined by the equation only. (In the case of the Painlevé-2 equation this is the finite complex plane.)

The modern framework for the study of the inverse monodromy problems is the theory of holomorphic vector bundles. We refer the reader to the survey papers [4] and [32] for all the details. The main idea is to identify the inverse monodromy problem for a given system of linear equations with the question of triviality for a certain holomorphic vector bundle over $\mathbb{C} P^{1} \times \mathcal{R}$, where $\mathcal{R}$ denotes a universal covering of the set of parameters of the linear system. By using powerful, although highly nontrivial, geometric tools of the theory of vector bundles, a negative answer to the surjectivity question for the monodromy map in the case of Fuchsian systems $(A(\lambda)$ has only simple poles and the monodromy set $\mathcal{S}$ coincides with the monodromy group - the 21st Hilbert problem) was obtained in [5]. The comprehensive analysis of additional conditions to be imposed on the monodromy data in order to ensure the solvability of the inverse monodromy problem for Fuchsian systems was done in [6] and [26]. In the case of a general linear system and for fixed monodromy data belonging to the image of the monodromy map, it was shown in 27] that the inverse generalized monodromy map is meromorphic as the function on the set $\mathcal{D}$ of deformation parameters. A similar result for Fuchsian systems was obtained in [29], and for a generic system with irregular singularities in [28, 30].11 Many important results concerning the solvability of the inverse monodromy problem for general linear systems were obtained in [2] 7, 25]. We refer the reader to the survey [7] for more on the holomorphic vector bundle approach to the inverse monodromy problem and on the history of the whole subject.

Another approach to the inverse monodromy problems is based on their interpretation as the Riemann-Hilbert factorization problems, and it invokes the analytic theory of Fredholm operators in order to establish the existence of the inverse monodromy maps under certain symmetry constraints on the monodromy data. This approach can also be used to prove that the inverse generalized monodromy map is meromorphic on the set of deformation parameters provided the monodromy data belong to the image of the monodromy map for at least one particular set of deformation parameters. 3 The method

\footnotetext{
${ }^{1}$ Perhaps, from the results of $27 \sqrt[30]{30}$, and $[28$ it is possible to deduce that the monodromy map is surjective in the generic case of rational coefficient matrix $A(\lambda)$; however, that much was not exactly stated in the papers cited.

${ }^{2}$ In principle, using certain topological arguments [11], one can drop this restriction on the monodromy data.
} 
was presented in [34, 8, 16], and [10]; in particular, it allows one to prove the Painlevé property for all the Painlevé transcendences 3 Also, the Riemann-Hilbert factorization setting made it possible to use the nonlinear steepest descent method of [12] for the asymptotic analysis of the inverse monodromy map and hence for the asymptotic analysis of the solutions of Painlevé equations (see [13] and also [21]).

The Fredholm theory of singular integral operators plays an important role in the vector bundle method as well; one needs it to analyze the local factorization problems near singular points (see 27, 30.) The geometric-topological arguments are used then to "glue" the solutions of local Riemann-Hilbert problems into a unique global solution. Therefore, in essence, the invariant geometric language of vector bundles is unavoidable for the rigorous analysis of the inverse monodromy problem and isomonodromy deformations in the case of general linear systems. At the same time, for specific linear systems related to the Painlevé equations, e.g., for system (1), it is possible to perform a rigorous study of the inverse problem on the basis of analytic considerations only.

Let us make a few additional remarks concerning the link between the Painlevé property of the solutions of isomonodromy deformation equations and the property to be meromorphic for the solutions of the related Riemann-Hilbert problems. If the Painlevé property is known a priori, the basic analytic properties of the direct monodromy map can be used to show that the solutions of the Riemann-Hilbert problem are meromorphic. This idea was used in [20, where the inverse generalized monodromy map was shown to exist and be meromorphic in a particular case (namely, $u=v, w=y$ ) of system (11); this was done by using the classical Birkhoff-Grothendieck theorem and by assuming the Painlevé property for the second Painlevé equation. However, this approach can hardly be extended to more general linear systems, because a direct proof of the statement that the solutions of general isomonodromy deformation equations are meromorphic, is overwhelmingly difficult. We accept the point of view on this matter that is usual for the theory of integrable system: the adequate way to analyze a global property (e.g., the Painlevé property) of the solutions of an integrable system is to invoke the associated Riemann-Hilbert problem. This is exactly the method by which the Painlevé property for general isomonodromy deformation equations was proved in [7] and [27-[28]. In a similar spirit (but not by the same technique), the results mentioned above on the explicit asymptotic connection formulas for Painlevé equations have also been obtained via the asymptotic analysis of the related Riemann-Hilbert problems.

In what follows, we present an elementary proof of the meromorphic solvability of the Riemann-Hilbert problem for a general case of system (1), for an arbitrary choice of monodromy data, and without any prior assumptions concerning the related system of isomonodromy deformations. As a corollary, we rigorously justify the monodromy data parametrization of the second and the thirty fourth Painlevé transcendents and prove that they are meromorphic. We call our proof "elementary" since we do not use (directly) either holomorphic vector bundles or the $L_{2}$-Fredholm theory. The basic technical ideas that we use instead are the following two: (i) the explicit solution of the model Riemann-Hilbert factorization problem near $\lambda=\infty$, and (ii) a generalization, due to B. Malgrange, of the classical Birkhoff-Grothendieck theorem to the case with the parameter. An elementary proof of this important theorem is presented in Appendix 1 ; this proof is modeled on that suggested in [7. We also make use of the uniform boundedness of the Cauchy operator in some natural Banach spaces of holomorphic functions (see Subsection 4.1 and Appendix 1).

\footnotetext{
${ }^{3}$ It should be noted that alternative methods to prove the Painlevé property for Painlevé equations, which do not use any relationship with the Riemann-Hilbert problem, were suggested in [23] and [9].
} 
We believe that the ever-widening application of the Painleve functions throughout increasingly numerous areas of analysis and mathematical physics, as well as the success of the Riemann-Hilbert approach in the asymptotic analysis of Painlevé transcendents, make it worth having a detailed proof of their basic analytic properties, as well as of the basic analytic properties of the solutions of the associated Riemann-Hilbert problems, accessible without bundle theory or the analytic Fredholm theory as prerequisites. At the same time, we hope that this paper will prepare the reader for the more advanced texts on the general Riemann-Hilbert-Birkhoff problem where the use of the invariant language is essential.

The second and third authors take the full responsibility for this Introduction and apologize for all possible flaws in its reference part.

\section{§2. Formulation of the Riemann-Hilbert problem and the main theorem}

We begin with the exact definition of the monodromy sets $\mathcal{M}$ and $\mathcal{S}$ and the monodromy maps (4) and (5). To this end, we need some basic facts concerning the solutions of system (1).

In accordance with the general theory of linear systems of ODEs with rational coefficients (see, e.g., 31] or [3]), system [1] has seven canonical solutions, $\Psi_{k}(\lambda), k=1, \ldots, 7$, which are uniquely determined by the asymptotic conditions

$$
\begin{array}{r}
\Psi_{k}(\lambda)=(I+o(\mathbb{1})) \exp \left\{-\frac{4}{3} i \lambda^{3} \sigma_{3}-i x \lambda \sigma_{3}-\nu \ln \lambda \sigma_{3}\right\}, \\
\lambda \rightarrow \infty, \quad \lambda \in \Omega_{k},
\end{array}
$$

where

$$
\nu=v w-u y
$$

is the formal monodromy exponent, and $\Omega_{k} \subset \mathbb{C}$ denotes the canonical sectors,

$$
\Omega_{k}=\left\{\lambda:-\frac{2 \pi}{3}+\frac{\pi k}{3}<\arg \lambda<\frac{\pi k}{3}\right\}, \quad k=1,2, \ldots, 7 .
$$

The branch of $\ln \lambda$ is fixed by the condition

$$
-\frac{2 \pi}{3}+\frac{\pi k}{3}<\arg \lambda<\frac{\pi k}{3} \quad \text { if } \lambda \in \Omega_{k} .
$$

The functions $\Psi_{k}$ satisfy the following analytic properties, which are the specifications for system (1) of the corresponding general facts of the theory of linear ODEs (see again [3] 31] and also [24).

(i) Each $\Psi_{k}(\lambda)$ is an entire function. Moreover, the functions $\Psi_{k}(\lambda)$ depend analytically on the parameters $u, v, w, y$, and $x$. In fact, if we denote

$$
\mathbf{a}:=(u, v, w, y, x) \in \mathcal{A},
$$

then each $\Psi_{k}(\lambda) \equiv \Psi_{k}(\lambda, \mathbf{a})$ is jointly analytic in $(\lambda, \mathbf{a})$. In other words,

$$
\Psi_{k}(\lambda, \mathbf{a}) \in H(\mathbb{C} \times \mathcal{A}) \equiv H\left(\mathbb{C} \times \mathbb{C}^{5}\right) .
$$

(ii) Each $\Psi_{k}(\lambda, \mathbf{a})$ is invertible for all $(\lambda, \mathbf{a})$, and

$$
\Psi_{k}^{-1}(\lambda, \mathbf{a}) \in H(\mathbb{C} \times \mathcal{A}) .
$$

In fact,

$$
\operatorname{det} \Psi_{k} \equiv 1 \text {. }
$$


(iii) Estimate (6) can be replaced by the full asymptotic series,

$$
\Psi_{k}(\lambda) \simeq \Psi_{f}(\lambda), \quad \lambda \rightarrow \infty, \lambda \in \Omega_{k},
$$

where $\Psi_{f}(\lambda)$ denotes the formal power series solution of (1),

$$
\Psi_{f}(\lambda)=\left(I+\sum_{j=1}^{\infty} \frac{m_{j}}{\lambda^{j}}\right) \exp \left\{-\frac{4}{3} i \lambda^{3} \sigma_{3}-i x \lambda \sigma_{3}-\nu \ln \lambda \sigma_{3}\right\} .
$$

The asymptotic expression (7) is uniform, i.e., for each positive number $l$, closed subsector $\Omega_{k}^{\prime} \subset \Omega_{k}$, and compact subset $\mathcal{K} \in \mathcal{A}$, there exists a constant $C>0$ such that

$$
\begin{array}{r}
\left|\Psi_{k}(\lambda) \exp \left\{\frac{4}{3} i \lambda^{3} \sigma_{3}+i x \lambda \sigma_{3}+\nu \ln \lambda \sigma_{3}\right\}-I-\sum_{j=1}^{l} \frac{m_{j}}{\lambda^{j}}\right| \leq \frac{C}{\lambda^{l+1}}, \\
(\lambda, \mathbf{a}) \in \Omega_{k}^{\prime} \times \mathcal{K} .
\end{array}
$$

(iv) The coefficients $m_{j}$ of the series (8) are entire functions of a. In fact, like the formal monodromy exponent $\nu$, all the $m_{j}$ are polynomials in $u, v, w, y$, and $x$, and they can be determined recursively (see, e.g., [24]). The first two coefficients are

where

$$
\begin{aligned}
m_{1} & =\frac{1}{2}\left(\begin{array}{cc}
-i d & u \\
v & i d
\end{array}\right), \\
m_{2} & =\frac{1}{8}\left(\begin{array}{cc}
u v-d^{2}-x \nu & 2 i w+2 i u d \\
-2 i y-2 i v d & u v-d^{2}+x \nu
\end{array}\right),
\end{aligned}
$$

(we recall that

$$
d=y w-x u v-u^{2} v^{2}
$$

$$
\nu=v w-u y
$$

is the formal monodromy exponent; see (6), (8) ).

(v) The asymptotic expansion (17) is termwise differentiable with respect to $\lambda$ and each of the coordinates of $\mathbf{a}$, i.e., with respect to $\lambda, u, v, w, y$, and $x$. The resulting asymptotic representations are uniform as well.

The Stokes matrices are defined by the equations

$$
S_{k}=\Psi_{k}^{-1}(\lambda) \Psi_{k+1}(\lambda), \quad k=1, \ldots, 6 .
$$

Since $\Psi_{k}(\lambda)$ and $\Psi_{k+1}(\lambda)$ are fundamental solutions of the same linear system, the matrices $S_{k}$ do not depend on $\lambda$. However, they depend on the coefficients of system (11), i.e.,

$$
S_{k} \equiv S_{k}(u, v, w, y, x) \equiv S_{k}(\mathbf{a})
$$

Moreover, properties (i) and (ii) of the canonical solutions imply

$$
S_{k}(\mathbf{a}) \in H(\mathcal{A}) \equiv H\left(\mathbb{C}^{5}\right) \text {. }
$$

Furthermore, the Stokes matrices have the following triangular structure:

$$
S_{2 l}=\left(\begin{array}{cc}
1 & s_{2 l} \\
0 & 1
\end{array}\right), \quad S_{2 l-1}=\left(\begin{array}{cc}
1 & 0 \\
s_{2 l-1} & 1
\end{array}\right), \quad l=1,2,3,
$$

and they satisfy the cyclic relation

$$
S_{1} S_{2} S_{3} S_{4} S_{5} S_{6}=e^{-2 \pi i \nu \sigma_{3}} .
$$

The indicated triangular structure of $S_{k}$ is a direct consequence of the fact that two different solutions of equation (1), i.e., $\Psi_{k}(\lambda)$ and $\Psi_{k+1}(\lambda)$, have the same exponential 
asymptotics (6) in the common sector $\Omega_{k} \cap \Omega_{k+1}$ (the Stokes phenomenon). The cyclic relation follows from the fact that each $\Psi_{k}(\lambda)$ is an entire function and, therefore,

$$
\Psi_{1}(\lambda)=\Psi_{1}\left(e^{-2 \pi i} \lambda\right)=\Psi_{7}(\lambda) e^{2 \pi i \nu \sigma_{3}}=\Psi_{1}(\lambda) S_{1} S_{2} S_{3} S_{4} S_{5} S_{6} e^{2 \pi i \nu \sigma_{3}} .
$$

It should also be mentioned that equation (14) indicates the triviality of the monodromy group of system (11).

We define the set $\mathcal{S}$ of monodromy data as the collection of the Stokes matrices and the formal monodromy exponent $\nu$, i.e.,

$$
\mathcal{S}:=\left\{\left(S_{1}, \ldots, S_{6} ; \nu\right): \text { the } S_{k} \text { satisfy (13), (14) }\right\} .
$$

We also define the set $\mathcal{M}$ of generalized monodromy data as the collection of the Stokes matrices and the two parameters $\nu$ and $x$, which determine the exponential factor in the asymptotics (7), i.e.,

$$
\mathcal{M}:=\left\{\left(S_{1}, \ldots, S_{6} ; \nu, x\right): \text { the } S_{k} \text { satisfy (13), (14) }\right\} .
$$

Accordingly, the generalized monodromy map (4) is defined by equations (12) and (11), and by the equation $x=a_{5}$. Observe that $\mathcal{M}$ is a complex manifold of dimension 5 , i.e.,

$$
\operatorname{dim}_{\mathbb{C}} \mathcal{M}=5=\operatorname{dim}_{\mathbb{C}} \mathcal{A} .
$$

Simple considerations show that the generalized monodromy map is an injection. Indeed (cf., e.g., 31]), if we assume that

$$
\nu(\mathbf{a})=\nu(\mathbf{b}), \quad x(\mathbf{a})=x(\mathbf{b})
$$

and

$$
S_{k}(\mathbf{a})=S_{k}(\mathbf{b}) \text { for all } k,
$$

then the matrix ratio $\Psi_{1}(\lambda, \mathbf{a}) \Psi_{1}^{-1}(\lambda, \mathbf{b})$ is an entire function of $\lambda$ that approaches the identity matrix as $\lambda$ approaches infinity. By the Liouville theorem, this implies that, in fact, this matrix ratio is the identity matrix. Hence,

$$
\Psi_{1}(\lambda, \mathbf{a})=\Psi_{1}(\lambda, \mathbf{b}) \text { for all } \lambda,
$$

and, in turn,

$$
A(\lambda, \mathbf{a})=A(\lambda, \mathbf{b}) \text { for all } \lambda \Longrightarrow \mathbf{a}=\mathbf{b} .
$$

As it has already been indicated, the questions about the surjectivity of the monodromy map and the analytic properties of the inverse generalized monodromy map with respect to the complex parameter $x$ constitute the Riemann-Hilbert inverse monodromy problem for system (11). In other words, we ask the following: Given a complex number $\nu$ and six $2 \times 2$ matrices $S_{k}$ satisfying equations (13) and (14), does there exist a linear system of class (11) that has the given data as its monodromy data? If the answer is in the affirmative, then what is the dependence of the inverse generalized monodromy map on the deformation parameter $x$ ? Our principal goal is to present a detailed proof of the following theorem, which answers these questions.

Theorem 1. Let $s \equiv\left(S_{1}, S_{2}, \ldots, S_{6} ; \nu\right)$ be monodromy data, i.e., a complex number $\nu$ and six $(2 \times 2)$-matrices satisfying equations (13) and (14). Then there exists a countable set $\Theta \equiv \Theta(s) \equiv\left\{x_{j} \equiv x_{j}(s)\right\} \subset \mathbb{C}, \quad x_{j} \rightarrow \infty$, and a unique matrix-valued function $\Psi(\lambda, x)$ with the following properties:

(i) $\Psi(\lambda, x)$ and $\Psi^{-1}(\lambda, x)$ are holomorphic in $\mathbb{C} \times(\mathbb{C} \backslash \Theta)$ and meromorphic along $\mathbb{C} \times \Theta$ (i.e., they have poles at $x_{j} \in \Theta$, and the coefficients of the corresponding Laurent series are holomorphic in $\mathbb{C}$ );

(ii) $\operatorname{det} \Psi(\lambda, x) \equiv 1$; 
(iii) for every $x \in \mathbb{C} \backslash \Theta$ we have

$$
\begin{aligned}
\Psi(\lambda, x) S_{1} \cdots S_{k-1} & \simeq\left(I+\sum_{j=1}^{\infty} \frac{m_{j}(x)}{\lambda^{j}}\right) e^{\left\{-\frac{4}{3} i \lambda^{3} \sigma_{3}-i x \lambda \sigma_{3}-\nu \ln \lambda \sigma_{3}\right\}}, \\
\lambda & \rightarrow \infty, \quad \lambda \in \Omega_{k}, \quad-\frac{2 \pi}{3}+\frac{\pi k}{3}<\arg \lambda<\frac{\pi k}{3},
\end{aligned}
$$

where the coefficients $m_{j}(x)$ are holomorphic in $\mathbb{C} \backslash \Theta$ and meromorphic in $\mathbb{C}$;

(iv) the asymptotic expression (15) is uniform, i.e., for each positive number l, closed subsector $\Omega_{k}^{\prime} \subset \Omega_{k}$, and compact subset $\mathcal{K} \subset \mathbb{C} \backslash \Theta$, there exists a constant $C>0$ such that

$$
\begin{array}{r}
\left|\Psi(\lambda, x) S_{1} \cdots S_{k-1} \exp \left\{\frac{4}{3} i \lambda^{3} \sigma_{3}+i x \lambda \sigma_{3}+\nu \ln \lambda \sigma_{3}\right\}-I-\sum_{j=1}^{l} \frac{m_{j}}{\lambda^{j}}\right| \leq \frac{C}{\lambda^{l+1}}, \\
(\lambda, x) \in \Omega_{k}^{\prime} \times \mathcal{K} ;
\end{array}
$$

(v) the asymptotic expression (15) is differentiable with respect to $x$ and $\lambda$.

Theorem 1 shows that for any given collection $s$ of monodromy data the RiemannHilbert-Birkhoff problem posed above is solvable meromorphically with respect to the parameter $x$. Indeed, put

$$
A(\lambda, x):=\frac{\partial \Psi(\lambda, x)}{\partial \lambda} \Psi^{-1}(\lambda, x)
$$

For every $x \in \mathbb{C} \backslash \Theta$, the matrix $A(\lambda, x)$ is an entire function of $\lambda$. Moreover, since the matrices $S_{k}$ do not depend on $\lambda$, we have

$$
\begin{aligned}
A(\lambda, x)= & \frac{\partial\left[\Psi(\lambda, x) S_{1} \cdots S_{k-1}\right]}{\partial \lambda}\left[\Psi(\lambda, x) S_{1} \cdots S_{k-1}\right]^{-1} \\
\simeq & \left(I+\sum_{j=1}^{\infty} \frac{m_{j}(x)}{\lambda^{j}}\right)\left(-4 i \lambda^{2} \sigma_{3}-i x \sigma_{3}-\frac{\nu}{\lambda} \sigma_{3}\right)\left(I+\sum_{j=1}^{\infty} \frac{m_{j}(x)}{\lambda^{j}}\right)^{-1} \\
& -\sum_{j=1}^{\infty} \frac{j m_{j}(x)}{\lambda^{j+1}}\left(I+\sum_{j=1}^{\infty} \frac{m_{j}(x)}{\lambda^{j}}\right)^{-1},
\end{aligned}
$$

as $\lambda \rightarrow \infty, \lambda \in \Omega_{k}$. This means that in the full neighborhood of $\lambda=\infty$ the following estimate is fulfilled:

$$
A(\lambda, x)=A_{2}(x) \lambda^{2}+A_{1}(x) \lambda+A_{0}(x)+O(1 / \lambda), \quad \lambda \rightarrow \infty,
$$

where

$$
\begin{gathered}
A_{2}(x)=-4 i \sigma_{3}, \quad A_{1}(x)=4 i\left[\sigma_{3}, m_{1}(x)\right], \\
A_{0}(x)=4 i\left[\sigma_{3}, m_{2}(x)\right]-i x \sigma_{3}-4 i\left[\sigma_{3}, m_{1}(x)\right] m_{1}(x),
\end{gathered}
$$

and $[$,$] denotes the usual matrix commutator. By the Liouville theorem, we conclude$ that in fact the exact equation

$$
A(\lambda, x)=A_{2}(x) \lambda^{2}+A_{1}(x) \lambda+A_{0}(x)
$$

occurs. Therefore, the function $\Psi(\lambda, x)$ is a fundamental solution of a linear system of class (1). Moreover, by the asymptotic formula (15), the corresponding monodromy data coincide with the given set $s$.

It should be noted that, as soon as the polynomial structure of $A(\lambda, x)$ is established, we can use the asymptotic relation (17) to express all the coefficients $m_{j}(x)$ in terms of the four scalar functions $u(x), v(x), w(x)$, and $y(x)$, which parametrize the matrix $A(\lambda, x)$ (in accordance with (22)). In particular, equations (19)-(1I) are fulfilled. 
In what follows, the function $\Psi(\lambda, x) \equiv \Psi(\lambda, x ; s)$ will be called the solution of the Riemann-Hilbert problem corresponding to the monodromy data $s=\left(S_{1}, \ldots, S_{6} ; \nu\right)$.

Remark 1 . Theorem 1 is new as stated, but may be viewed properly as a specification and refinement of the results of [7, 16, 27, 28, 30, 4 The elementary proof of the theorem we present in $\S 4$ does not use the results of those papers.

\section{§3. Isomonodromy deformations. The Painlevé Property. The Second PAINLEVÉ EQUATION}

This section is an adaptation of the general theory of [14, 24 to system (1).

From equations (18), (19) it follows that the parameters $u, v, w$, and $y$ corresponding to the set

$$
s=\left(S_{1}, \ldots, S_{6} ; \nu\right)
$$

of monodromy data via the solution of the Riemann-Hilbert problem are given by the equations

$$
\begin{gathered}
u=2\left(m_{1}(x)\right)_{12}, \quad v=2\left(m_{1}(x)\right)_{21}, \\
w=-4 i\left(m_{2}(x)\right)_{12}+2 i u\left(m_{1}(x)\right)_{22}, \\
y=4 i\left(m_{2}(x)\right)_{21}-2 i v\left(m_{1}(x)\right)_{11},
\end{gathered}
$$

and therefore are meromorphic functions of $x$ with the set $\Theta \equiv \Theta(s)$ as the set of their poles. These functions have an important meaning; indeed, equations (21)-(23) determine the curve

$$
\mathbf{a}(x)=(u(x), v(x), w(x), y(x), x)
$$

in the space $\mathcal{A}$ of singular data such that the corresponding monodromy data are the same (coincide with the given set $s$ ) along the entire curve. In other words, these equations determine an isomonodromy deformation of system (11). The following theorem identifies the curve $\mathbf{a}(x)$ as a solution of a certain nonlinear system of ODEs; this system describes the isomonodromy deformations of the linear equation (1).

Theorem 2 (cf. [14, 20, 24]). Let $\Psi(\lambda, x)$ be the solution of the Riemann-HilbertBirkhoff problem corresponding to the set of monodromy data $s=\left(S_{1}, \ldots, S_{6} ; \nu\right)$, and let the (meromorphic) functions $u(x), v(x), w(x)$, and $y(x)$ be defined in accordance with (21) - 231). Ther

$$
w=u_{x}, \quad y=v_{x}
$$

and the functions $u(x)$ and $v(x)$ satisfy the following system of ODEs:

$$
\left\{\begin{array}{l}
u_{x x}=x u+2 u^{2} v, \\
v_{x x}=x v+2 v^{2} u .
\end{array}\right.
$$

Proof. Consider the $x$-logarithmic derivative of the function $\Psi(\lambda, x)$,

$$
U(\lambda, x):=\frac{\partial \Psi(\lambda, x)}{\partial x} \Psi^{-1}(\lambda, x) \equiv \Psi_{x}(\lambda, x) \Psi^{-1}(\lambda, x) .
$$

\footnotetext{
${ }^{4}$ The theorem also follows from the results of [20], which are based on the a priori assumption of the Painlevé property for the second Painlevé equation.

${ }^{5}$ We use the notation $f_{x} \equiv \frac{d f}{d x}$.
} 
Using this time the fact that the matrices $S_{k}$ and the monodromy exponent $\nu$ do not depend on $x$, as in (17)-(20) we find that

$$
\begin{aligned}
U(\lambda, x) \simeq & \left(I+\sum_{j=1}^{\infty} \frac{m_{j}(x)}{\lambda^{j}}\right)\left(-i \lambda \sigma_{3}\right)\left(I+\sum_{j=1}^{\infty} \frac{m_{j}(x)}{\lambda^{j}}\right)^{-1} \\
& +\sum_{j=1}^{\infty} \frac{m_{j x}(x)}{\lambda^{j}}\left(I+\sum_{j=1}^{\infty} \frac{m_{j}(x)}{\lambda^{j}}\right)^{-1}, \quad \lambda \rightarrow \infty,
\end{aligned}
$$

for every $x \in \mathbb{C} \backslash \Theta$. Like the $\lambda$-derivative $A(\lambda, x)$, the function $U(\lambda, x)$ is an entire function of $\lambda$. Therefore, the asymptotic relation (27) implies the exact equation

$$
U(\lambda, x)=U_{1}(x) \lambda+U_{0}(x),
$$

where

$$
U_{1}(x)=-i \sigma_{3}
$$

and

$$
U_{0}(x)=i\left[\sigma_{3}, m_{1}(x)\right]=\left(\begin{array}{cc}
0 & i u(x) \\
-i v(x) & 0
\end{array}\right) .
$$

As soon as the polynomial structure of $U(\lambda, x)$ is established, we can use the asymptotic formula (27) to obtain a series of identities involving the coefficient matrices $m_{j}(x)$. In particular, comparing the terms of order $\lambda^{-1}$, we arrive at the relation

$$
0=i\left[\sigma_{3}, m_{2}(x)\right]-i\left[\sigma_{3}, m_{1}(x)\right] m_{1}(x)+m_{1 x}(x) .
$$

Together with (19), this implies the formula

$$
A_{0}=-i x \sigma_{3}-4 m_{1 x}
$$

and equations (24) follow by (21).

To derive the nonlinear system (25), we take a new look at equations (16) and (26). Indeed, consider the linear system

$$
\left\{\begin{array}{l}
\Psi_{\lambda}=A(\lambda) \Psi \\
\Psi_{x}=U(\lambda) \Psi
\end{array}\right.
$$

where the polynomial matrices $A(\lambda) \equiv A(\lambda, x)$ and $U(\lambda) \equiv U(\lambda, x)$ are given by the equations

$$
\begin{aligned}
A(\lambda) & =A_{2} \lambda^{2}+A_{1} \lambda+A_{0} \\
& =-4 i \lambda^{2} \sigma_{3}+4 i \lambda\left(\begin{array}{cc}
0 & u \\
-v & 0
\end{array}\right)+\left(\begin{array}{cc}
-i x-2 i u v & -2 u_{x} \\
-2 v_{x} & i x+2 i u v
\end{array}\right)
\end{aligned}
$$

and

$$
U(\lambda)=U_{1} \lambda+U_{0}=-i \lambda \sigma_{3}+\left(\begin{array}{cc}
0 & i u \\
-i v & 0
\end{array}\right) .
$$

Then, we can interpret (16) and (26) as the following statement: Let $\Psi(\lambda, x)$ be the solution of the Riemann-Hilbert-Birkhoff problem, and let the scalar functional parameters $u(x)$ and $v(x)$ in (32) and (331) be defined in accordance with (21). Then system (31) is compatible and the function $\Psi(\lambda, x)$ is its fundamental solution. Observe that the compatibility condition $\Psi_{\lambda x}=\Psi_{x \lambda}$ yields the following relation on the coefficient matrices of the linear system (31):

$$
U_{\lambda}(\lambda)-A_{x}(\lambda)=[A(\lambda), U(\lambda)] \text { identically in } \lambda .
$$


A straightforward calculation shows that the nonlinear system (25) is equivalent to relation (34) provided $A(\lambda)$ and $U(\lambda)$ are given by equations (32) and (33), respectively. This completes the proof of the theorem.

The next theorem shows that any solution of (25) represents an isomonodromy deformation of system (1).

Theorem 3 (cf. [14, 20, 24]). Let $J \subset \mathbb{R}$ be an interval and suppose that $u(x)$ and $v(x)$ both belong to $C^{2}(J)$ and satisfy system (25). Then the equation

$$
\mathbf{a}=\left(u(x), v(x), u_{x}(x), v_{x}(x), x\right)
$$

determines an isomonodromic curve in the space $\mathcal{A}$ of singular data of system (10).

Proof. Again, we shall use the equivalence of the nonlinear system 25] to the compatibility condition (34) of the overdetermined linear system (31). Let $u(x)$ and $v(x)$ be functions satisfying the conditions of the theorem. Consider the linear system (1) with

$$
A(\lambda)=A(\lambda, x) \equiv A(\lambda, \mathbf{a}(x)),
$$

where

$$
\mathbf{a}(x):=\left(u(x), v(x), u_{x}(x), v_{x}(x), x\right),
$$

and let

$$
\Psi_{k}(\lambda, x) \equiv \Psi_{k}(\lambda, \mathbf{a}(x))
$$

be the corresponding canonical solutions. Note that the $\Psi_{k}(\lambda, x)$ are twice continuously differentiable with respect to $x$ (this follows from property (i) of the canonical solutions). It should also be noted that the combination $u_{x} v-u v_{x}$ is the first integral of system (25) (a simple direct calculation). Therefore, the formal monodromy exponent associated with $\Psi_{k}(\lambda, x)$, namely,

$$
\nu=v w-u y=u_{x} v-u v_{x},
$$

is a constant, i.e.,

$$
\frac{d \nu}{d x} \equiv 0
$$

Now we claim that for every $k$ and $\lambda$ we have

$$
\frac{\partial}{\partial x} \Psi_{k}(\lambda, x)=U(\lambda, x) \Psi(\lambda, x),
$$

where $U(\lambda, x)$ is defined by (33). Observe that the statement of the theorem is a simple consequence of this equation. Indeed, since (37) is followed by the relation

$$
\frac{\partial}{\partial x} \Psi_{k}^{-1}(\lambda, x)=-\Psi_{k}^{-1}(\lambda, x) U(\lambda, x),
$$

we have

$$
\begin{aligned}
\frac{d S_{k}(x)}{d x} & =\frac{\partial}{\partial x}\left(\Psi_{k}^{-1}(\lambda, x) \Psi_{k+1}(\lambda, x)\right) \\
& =-\Psi_{k}^{-1}(\lambda, x) U(\lambda, x) \Psi_{k+1}(\lambda, x)+\Psi_{k}^{-1}(\lambda, x) U(\lambda, x) \Psi_{k+1}(\lambda, x)=0 .
\end{aligned}
$$

Together with (36), this implies that the curve (35) is isomonodromic.

To verify equation (37), put

$$
F(\lambda, x):=\frac{\partial}{\partial x} \Psi_{k}(\lambda, x)-U(\lambda, x) \Psi_{k}(\lambda, x) .
$$


By (21) and (36), we see at once that 6

$$
F(\lambda, x) e^{\theta(\lambda, x) \sigma_{3}}=O(1 / \lambda) \quad \text { as } \lambda \rightarrow \infty, \lambda \in \Omega_{k},
$$

for every $x \in J$, where we have introduced the notation

$$
\theta(\lambda, x)=i \frac{4}{3} \lambda^{3}+i x \lambda+\nu \ln \lambda .
$$

We must show that, actually, $F(\lambda, x) \equiv 0$.

Using (34) (which is true because $u$ and $v$ satisfy (25)), we can easily check that the matrix-valued function $F(\lambda, x)$ satisfies system (11) for every $x$. It follows that there exists a matrix $C \equiv C(x)$ independent of $\lambda$ such that

$$
F(\lambda, x)=\Psi_{k}(\lambda, x) C(x) .
$$

From estimates (17) and (38) we conclude that, for every $x \in J$,

$$
e^{-\theta(\lambda, x) \sigma_{3}} C(x) e^{\theta(\lambda, x) \sigma_{3}}=O(1 / \lambda) \quad \text { as } \lambda \rightarrow \infty, \lambda \in \Omega_{k} .
$$

This estimate implies immediately that the diagonal entries of $C(x)$ vanish, i.e., $C_{11}(x)=$ $C_{22}(x) \equiv 0$. Then, assuming

$$
\frac{\pi}{3}(k-1)<\arg \lambda<\frac{\pi}{3} k
$$

in (39), we conclude that either $C_{12}(x)$ or $C_{21}(x)$ must be identically zero (depending on the parity of $k$ ). The remaining off-diagonal entry vanishes under the assumption that

$$
\frac{\pi}{3}(k-2)<\arg \lambda<\frac{\pi}{3}(k-1)
$$

in (39). Hence, the matrix $C(x)$ is zero for all $x$, and (37) follows. The proof of the theorem is complete.

Because of the uniqueness of a local solution of the Cauchy problem for system (25) and the uniqueness of the solution of the Riemann-Hilbert problem, we arrive at once at the following two important corollaries of Theorems 2 and 3.

Corollary 1 (The Painlevé property). Suppose that $C^{2}$-functions $u(x)$ and $v(x)$ satisfy the isomonodromy deformation equations (25). Then they admit meromorphic continuation to the entire complex $x$-plane.

Corollary 2 (Monodromy parametrization of the solution set). The map

$$
\{\text { solutions of }(\underline{25})\} \mapsto \mathcal{S}
$$

is a bijection; hence, the notation

$$
u(x) \equiv u(x ; s), \quad v(x) \equiv v(x ; s)
$$

for the solutions of (25) is justified. Given $s \in \mathcal{S}$, the functions $u(x ; s)$ and $v(x ; s)$ are meromorphic, and $\Theta(s)$ is the set of their (first order) poles.

In fact, the nonlinear system (25) is closely related to the classical list of Painlevé equations. Indeed, it can be observed that, under the reduction relation

$$
u=v,
$$

system (25) becomes a particular case of the second Painlevé equation

$$
u_{x x}=x u+2 u^{3} .
$$

\footnotetext{
${ }^{6}$ We recall that, by property (v) of the canonical solutions, the asymptotic expression (7) is $x$ differentiable.
} 
In terms of monodromy data, the reduction (40) is equivalent to the conditions

$$
\nu=0, \quad s_{k+3}=-s_{k}, \quad k=1,2,3
$$

(see, e.g., [19] and also Appendix 2); in particular, the cyclic equation (14) transforms into a single scalar equation, namely,

$$
s_{1}-s_{2}+s_{3}+s_{1} s_{2} s_{3}=0 .
$$

In its turn, this implies that, in the case of the second Painlevé equation (41), the set $\mathcal{S}$ of monodromy data becomes a two-dimensional algebraic variety:

$$
\mathcal{S}_{p 2}^{0}=\left\{s \equiv\left(s_{1}, s_{2}, s_{3}\right) \in \mathbb{C}^{3}: s_{1}-s_{2}+s_{3}+s_{1} s_{2} s_{3}=0\right\} .
$$

From Corollaries 2 and 3 we deduce the respective statements concerning the Painlevé equation (41).

Corollary $\mathbf{1}^{0}$ (The Painlevé property). Suppose that a $C^{2}$-function $u(x)$ satisfies the second Painlevé equation (41). Then it admits meromorphic continuation to the entire complex $x$-plane.

Corollary $\mathbf{2}^{0}$ (Monodromy parametrization of the Painlevé set). The map

$$
\{\text { solutions of } P 2\} \mapsto \mathcal{S}_{p 2}^{0}
$$

is a bijection; hence, the notation

$$
u(x) \equiv u(x ; s),
$$

for the solutions of the Painlevé equation is justified. Given $s \in \mathcal{S}_{p 2}^{0}$, the function $u(x ; s)$ is meromorphic, and $\Theta(s)$ is the set of its (first order) poles.

In Appendix 2, more facts concerning the relationship of the system (25) with Painlevé equations are collected. In particular, there we describe links to the full Painlevé-2 equation and to the Painlevé-34 equation.

\section{$\S 4$. Proof of Theorem 1}

We split the proof into three steps. At the first step, we establish the existence of a solution local both in $\lambda$ and in $x$ for the Riemann-Hilbert problem in question. Specifically, we prove that, given a neighborhood $\Omega$ of $\lambda=\infty$ and a disk $D$ on the $x$ plane, there exists a matrix-valued function (denoted by $M(\lambda, x)$ ) that is holomorphically invertible in $\Omega \times D$ and satisfies the asymptotic condition (15) with all $m_{j}(x)$ holomorphic in $D$ (see Theorem 4 below). We note that, were it not for our special interest in the $x$-dependence, the result would follow from the Sibuya theorem 31] concerning the local solvability of the general Riemann-Hilbert-Birkhoff problem for a linear system near its irregular singularity. Therefore, we call this step the Sibuya theorem with a parameter. The principal construction used at this step is the introduction of the explicit model functions $g_{k}(\lambda, x)$ such that each of them factorizes the corresponding Stokes matrix $S_{k}$. Essentially, it is this technique, followed by an application of the Birkhoff-Grothendieck theorem (the second step), that allows us to avoid any use of the $L_{2}$-Fredholm theory of singular integral operators, which was a principal tool in the papers [8] and [16] mentioned before.

At the second step, we apply the classical Birkhoff-Grothendieck theorem (also generalized to the case with a parameter) to the function $M(\lambda, x)$ and obtain a solution global in $\lambda$, but still local in $x$, for the original Riemann-Hilbert problem (see Theorem 6 below). Since this is the most important step, for the reader's convenience we decided to present a detailed proof of the Birkhoff-Grothendieck theorem with parameter in Appendix 1; this proof follows the pattern of [7]. 
Finally, at the third step we cover the $x$-plane by a countable number of small disks and glue up the corresponding local solutions into a single solution (global both in $\lambda$ and in $x$ ) of the inverse monodromy problem in question, which completes the proof of Theorem 1.

Before we proceed, we make a technical assumption concerning the formal monodromy exponent $\nu$. We assume that

$$
\nu \notin \mathbb{Z} \backslash\{0\} .
$$

The following lemma shows that it indeed suffices to prove Theorem 1 under this restriction.

Lemma 1. Let $\Psi(\lambda, x)$ be the solution of the Riemann-Hilbert problem with monodromy data $s=\left(S_{1}, \ldots, S_{6} ; \nu\right)$, and let $u(x)$ and $v(x)$ be the corresponding functional parameters on the manifold $\mathcal{A}$ of singular data. Then the functions

$$
\hat{\Psi}(\lambda, x):=\left(\begin{array}{cc}
\lambda+u_{x} / 2 i u & -u / 2 \\
2 / u & 0
\end{array}\right) \Psi(\lambda, x)
$$

and

$$
\tilde{\Psi}(\lambda, x):=\left(\begin{array}{cc}
0 & 2 / v \\
-v / 2 & \lambda-v_{x} / 2 i v
\end{array}\right) \Psi(\lambda, x)
$$

solve the Riemann-Hilbert problems with the monodromy data

$$
\hat{s}=\left(S_{1}, \ldots, S_{6} ; \nu-1\right)
$$

and

$$
\tilde{s}=\left(S_{1}, \ldots, S_{6} ; \nu+1\right),
$$

respectively.

Note that only the asymptotic property (iii) needs a proof. The proof can be obtained by direct calculation with the help of relations (21) - 23) between the matrix coefficients $m_{j}$ of the asymptotic series for $\Psi$ and the functions $u, v, w=u_{x}$, and $y=v_{x}$ ఫ

4.1. The Sibuya theorem with a parameter. In this section, as well as in the rest of the paper, we accept the following notation. For each set $\mathbb{M} \subset \mathbb{C}^{N}$ with nonempty interior, we denote by $H(\mathbb{M})$ the Banach space of $2 \times 2$ matrix-valued functions holomorphic in the interior of $\mathbb{M}$ and continuous in $\overline{\mathbb{M}}$. Next, we denote by $H^{0}(\mathbb{M})$ the subspace of $H(\mathbb{M})$ consisting of holomorphically invertible matrix functions. Such functions are also said to be holomorphic in $\mathbb{M}$ and holomorphically invertible in $\mathbb{M}$, respectively.

Now, let

$$
\Omega_{\rho}=\{\lambda \in \mathbb{C}:|\lambda|>\rho\}
$$

\footnotetext{
${ }^{7}$ The transformations $\Psi \mapsto \hat{\Psi}$ and $\Psi \mapsto \tilde{\Psi}$ are examples (found by A. Kitaev) of the Schlesinger transformations (see, e.g., 24). They induce the Bäklund transformations

$$
(u, v) \mapsto\left(-\frac{u}{4}\left(u v+x-u_{x}^{2} / u^{2}\right), \frac{4}{u}\right),
$$

and

$$
(u, v) \mapsto\left(\frac{4}{v},-\frac{v}{4}\left(u v+x-v_{x}^{2} / v^{2}\right)\right),
$$

on the solution space for system [25.
} 


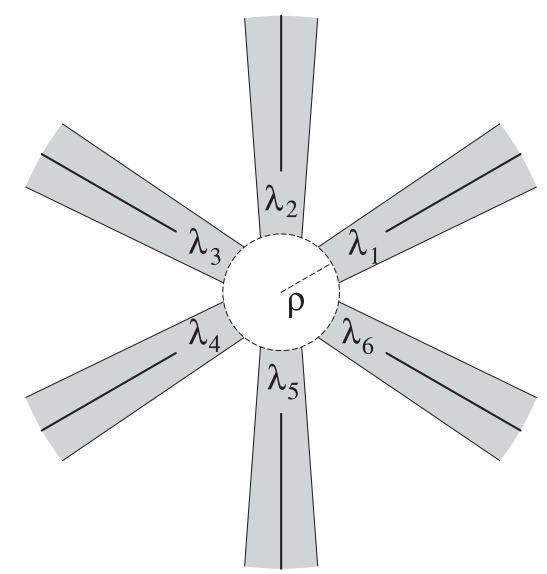

FiguRE 1. The contours $\Gamma_{k}^{\rho}$ and the sets $\Omega_{k}^{\rho}$.

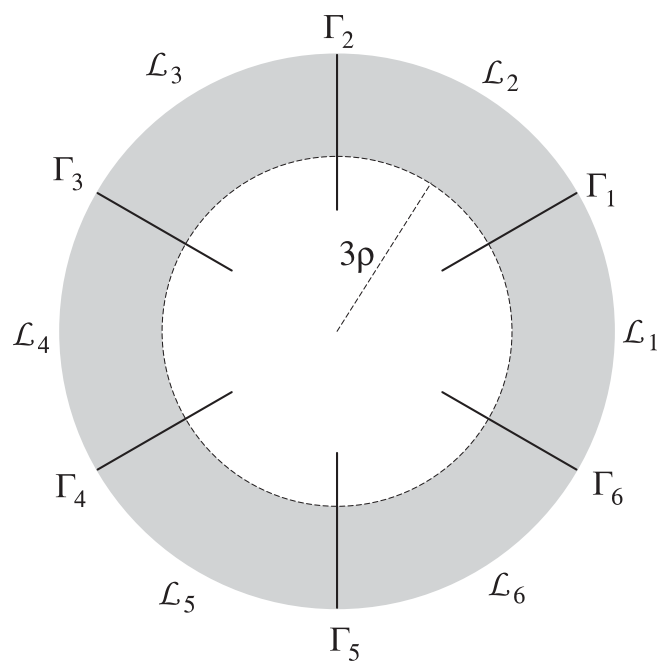

Figure 2. The sectors $\mathcal{L}_{k}$.

be a neighborhood of $\lambda=\infty$. We define the following subsets of $\Omega_{\rho}$ :

$$
\begin{aligned}
\Gamma_{k}^{\rho} & =\left\{\lambda \in \mathbb{C}:|\lambda| \geq 2 \rho, \arg \lambda=\frac{2 k-1}{6} \pi\right\}, \quad k=1, \ldots, 6 ; \\
\Omega_{k}^{\rho} & =\left\{\lambda \in \mathbb{C}:|\lambda|>\rho,\left|\arg \lambda-\frac{2 k-1}{6} \pi\right|<\varepsilon\right\} ; \\
\mathcal{L}_{k} & =\left\{\lambda \in \mathbb{C}:|\lambda| \geq 3 \rho,\left|\arg \lambda-\frac{k-1}{3} \pi\right| \leq \frac{\pi}{6}\right\}, \quad k=1, \ldots, 6 .
\end{aligned}
$$

We assume that the contours $\Gamma_{k}^{\rho}$ are oriented towards the point at infinity, and $\varepsilon$ is a sufficiently small positive number such that $\Omega_{k}^{\rho} \cap \Omega_{k+1}^{\rho}=\varnothing$. At the moment, we do not impose any restrictions on the positive number $\rho$. The contours $\Gamma_{k}^{\rho}$ and the sets $\Omega_{k}^{\rho}$ are depicted in Figure 1. The sectors $\mathcal{L}_{k}$ are presented in Figure 2.

Our goal in this section is to prove the following theorem. 
Theorem 4 (The Sibuya theorem with a parameter). Let $s=\left(S_{1}, \ldots, S_{6} ; \nu\right) \in \mathcal{S}$ be given monodromy data, and let $\mathcal{K}$ be a closed disk on the complex $x$-plane. Then there exists $\rho_{0} \equiv \rho_{0}(\mathcal{K})$ and a matrix-valued function $M(\lambda, x)$ such that for all $\rho \geq \rho_{0}$ the following statements are true.

1. $M(\lambda, x) \in H^{0}\left(\Omega_{3 \rho} \times \mathcal{K}\right)$.

2. For every $x \in \mathcal{K}$, we have

$$
\begin{aligned}
M(\lambda, x) S_{1} \cdots S_{k-1} & \left(I-\sum_{j=1}^{\infty} \frac{m_{j}^{0}(x)}{\lambda^{j}}\right) e^{\left\{-\frac{4}{3} i \lambda^{3} \sigma_{3}-i x \lambda \sigma_{3}-\nu \ln \lambda \sigma_{3}\right\}}, \\
& \lambda \rightarrow \infty, \quad \lambda \in \mathcal{L}_{k}, \quad\left|\arg \lambda-\frac{k-1}{3} \pi\right| \leq \frac{\pi}{6},
\end{aligned}
$$

where the coefficients $m_{j}^{0}(x)$ are holomorphic in $\mathcal{K}$.

3. The asymptotic expression (45) is uniform, i.e., for any positive number $l$, there exists a constant $C>0$ such that

$$
\begin{array}{r}
\left|M(\lambda, x) S_{1} \cdots S_{k-1} \exp \left\{\frac{4}{3} i \lambda^{3} \sigma_{3}+i x \lambda \sigma_{3}+\nu \ln \lambda \sigma_{3}\right\}-I-\sum_{j=1}^{l} \frac{m_{j}^{0}}{\lambda^{j}}\right| \leq \frac{C}{\lambda^{l+1}}, \\
(\lambda, x) \in \mathcal{L}_{k} \times \mathcal{K} .
\end{array}
$$

4. The asymptotic expression (45) is differentiable with respect to both $x$ and $\lambda$.

Remark 2. Because of the "correct" triangular structure of the Stokes matrices $S_{k}$, the asymptotic condition (45) implies that it is actually fulfilled in the open sectors $\Omega_{k}$ defined in (6). Indeed, for every positive number $l$ and closed subsector $\Omega_{k}^{\prime} \subset \Omega_{k}$ there exists a constant $C>0$ such that

$$
\begin{array}{r}
\left|M(\lambda, x) S_{1} \cdots S_{k-1} \exp \left\{\frac{4}{3} i \lambda^{3} \sigma_{3}+i x \lambda \sigma_{3}+\nu \ln \lambda \sigma_{3}\right\}-I-\sum_{j=1}^{l} \frac{m_{j}^{0}}{\lambda^{j}}\right| \leq \frac{C}{\lambda^{l+1}}, \\
(\lambda, x) \in \Omega_{k}^{\prime} \times \mathcal{K} .
\end{array}
$$

In an equivalent way, Theorem 4 can be reformulated as follows.

Theorem $4^{\prime}$. Let $s=\left(S_{1}, \ldots, S_{6} ; \nu\right) \in \mathcal{S}$ be given monodromy data, and let $\mathcal{K}$ be a closed disk on the complex $x$-plane. Then there exists $\rho_{0} \equiv \rho_{0}(\mathcal{K})$ and a collection of six matrix-valued functions $Y_{k}(\lambda, x), k=1, \ldots, 6$, such that for all $\rho \geq \rho_{0}$ the following statements are true.

1. $Y_{k}(\lambda, x) \in H^{0}\left(\mathcal{L}_{k} \times \mathcal{K}\right)$.

2. $Y_{k+1}(\lambda, x)=Y_{k}(\lambda, x) G_{k}(\lambda, x), \lambda \in \Gamma_{k}^{\rho} \cap\{|\lambda| \geq 3 \rho\}, k=1, \ldots, 6$, where

$$
\begin{aligned}
G_{k}(\lambda, x)=e^{-\theta(\lambda, x) \sigma_{3}} S_{k} e^{\theta(\lambda, x) \sigma_{3}}, \quad \theta(\lambda, x) & =i \frac{4}{3} \lambda^{3}+i x \lambda+\nu \ln \lambda, \\
\arg \lambda & =\frac{2 k-1}{6} \pi \quad \text { if } \lambda \in \Gamma_{k}^{\rho},
\end{aligned}
$$

and we put $Y_{7}(\lambda, x) \equiv Y_{1}(\lambda, x)$ 8

3. For every $x \in \mathcal{K}$ and $k=1, \ldots, 6$ we have

$$
\begin{aligned}
Y_{k}(\lambda, x) & \simeq I+\sum_{j=1}^{\infty} \frac{m_{j}^{0}(x)}{\lambda^{j}}, \\
\lambda & \rightarrow \infty, \quad \lambda \in \mathcal{L}_{k},
\end{aligned}
$$

\footnotetext{
${ }^{8}$ We emphasize that the sectors $\mathcal{L}_{k}$ are viewed as subsets of $\mathbb{C}$ but not of the universal covering of $\mathbb{C} \backslash\{0\}$.
} 
where the coefficients $m_{j}^{0}(x)$ are holomorphic in $\mathcal{K}$.

4. The asymptotic expression (47) is uniform, i.e., for each positive number $l$ there exists a constant $C>0$ such that

$$
\left|Y_{k}(\lambda, x)-I-\sum_{j=1}^{l} \frac{m_{j}^{0}}{\lambda^{j}}\right| \leq \frac{C}{\lambda^{l+1}} \quad \text { for all }(\lambda, x) \in \mathcal{L}_{k} \times \mathcal{K} \text { and all } k .
$$

5. The asymptotic expression (47) is differentiable with respect to both $x$ and $\lambda$.

To see that Theorem $4^{\prime}$ implies Theorem 4, we define a function $M(\lambda, x)$ by the equations

$$
\begin{aligned}
& \left.M(\lambda, x)\right|_{\mathcal{L}_{1}}=Y_{1}(\lambda, x) e^{-\theta(\lambda, x) \sigma_{3}}, \\
& \left.M(\lambda, x)\right|_{\mathcal{L}_{k}}=Y_{k}(\lambda, x) e^{-\theta(\lambda, x) \sigma_{3}} S_{k-1} \cdots S_{1}, \quad k>1,
\end{aligned}
$$

where the branch of $\ln \lambda$ is fixed by the condition

$$
\left|\arg \lambda-\frac{k-1}{3} \pi\right| \leq \frac{\pi}{6} \quad \text { if } \lambda \in \mathcal{L}_{k}
$$

The function $M(\lambda, x)$ defined in this way satisfies all the statements of Theorem 3 . Indeed, because of property 2 of $Y_{k}(\lambda, x)$ and the cyclic constraint (14) on $S_{k}$, the function $M(\lambda, x)$ has no jumps across any of $\Gamma_{k}^{\rho}$; hence, it is holomorphic in the entire $\Omega_{3 \rho}$. The rest of the properties announced in Theorem 4 follow directly from the respective properties of the functions $Y_{k}(\lambda, x)$.

In what follows, we prove Theorem $4^{\prime}$. Our approach is based on the replacement of the jump conditions posed on the rays $\Gamma_{k}^{\rho}$ by some equivalent jump conditions posed on horseshoe shape domains around the rays. To this end, we introduce the following covering of the neighborhood $\Omega_{\rho}$ :

$$
\Omega_{\rho} \subset \Omega_{+} \cup \Omega_{-}
$$

where the sets $\Omega_{+}$and $\Omega_{-}$are as depicted in Figure 3 and Figure 4 , respectively. The characteristic properties of $\Omega_{+}$and $\Omega_{-}$are as follows.

- The set $\Omega_{+}$is obtained from the complex plane $\mathbb{C}$ by deleting a collection of thin neighborhoods $\Omega_{+}^{k}$ of the contours $\Gamma_{k}^{\rho}$. The closure of the neighborhood $\Omega_{+}^{k}$ is a subset of $\Omega_{k}^{\rho}$. In other words,

$$
\Omega_{+}=\mathbb{C} \backslash \bigcup_{j=1}^{6} \overline{\Omega_{+}^{k}}, \quad \Gamma_{k}^{\rho} \subset \Omega_{+}^{k}, \quad \overline{\Omega_{+}^{k}} \subset \Omega_{k}^{\rho} .
$$

- The set $\Omega_{-}$is a union of (slightly bigger) neighborhoods $\Omega_{-}^{k}$ of the contours $\Gamma_{k}^{\rho}$, whose closures are also subsets of the respective $\Omega_{k}^{\rho}$. In other words,

$$
\Omega_{-}=\bigcup_{k=1}^{6} \Omega_{-}^{k}, \quad \Gamma_{k}^{\rho} \subset \Omega_{+}^{k}, \quad \overline{\Omega_{+}^{k}} \subset \Omega_{-}^{k}, \quad \overline{\Omega_{-}^{k}} \subset \Omega_{k}^{\rho} .
$$

- Let $\gamma^{+}:=\partial \Omega_{+}$be the boundary of $\Omega_{+}$. Then

$$
\gamma^{+}=\bigcup_{k} \gamma_{k}^{+}, \quad \gamma_{k}^{+}=-\partial \Omega_{+}^{k}
$$

and we assume that each $\gamma_{k}^{+}$is a simple analytic curve that encircles the ray $\Gamma_{k}^{\rho}$ and is asymptotic to the rays $\Gamma_{(k ; \pm \varepsilon / 3)}$, where

$$
\Gamma_{(k ; \delta)}:=\left\{\lambda \in \mathbb{C}: \arg \lambda-\frac{2 k-1}{6} \pi=\delta\right\} .
$$




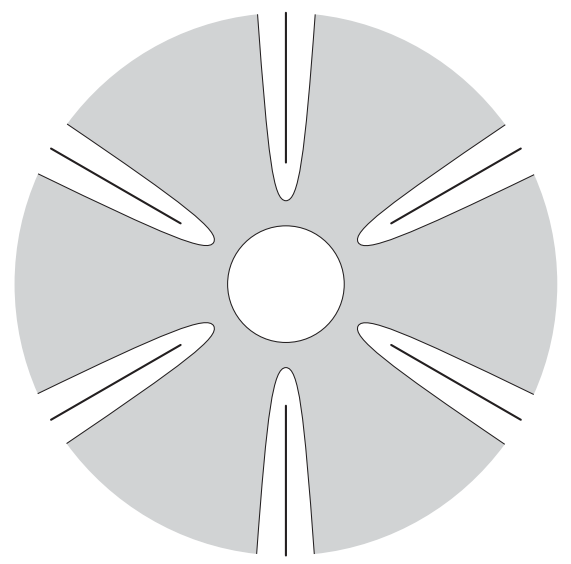

Figure 3 . The set $\Omega_{+}=\mathbb{C} \backslash \bigcup_{j=1}^{6} \overline{\Omega_{+}^{k}}$.

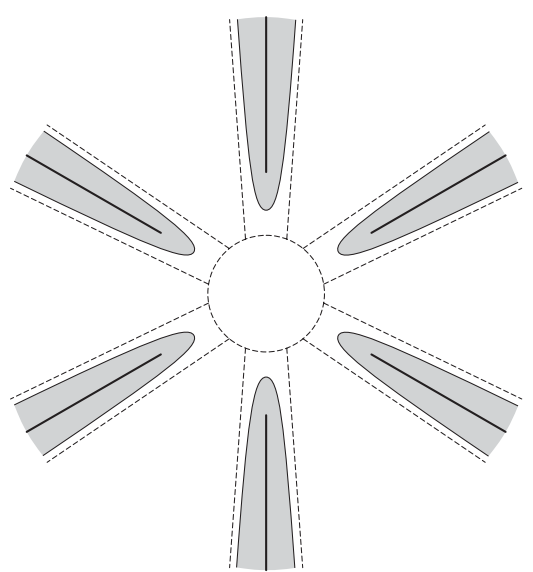

Figure 4 . The set $\Omega_{-}=\bigcup_{k=1}^{6} \Omega_{-}^{k}$.

- Let $\gamma^{-}:=\partial \Omega_{-}$be the boundary of $\Omega_{-}$. Then

$$
\gamma^{-}=\bigcup_{k} \gamma_{k}^{-}, \quad \gamma_{k}^{-}=\partial \Omega_{-}^{k},
$$

and we assume that each $\gamma_{k}^{-}$is a simple analytic curve that encircles the ray $\Gamma_{k}^{\rho}$ and is asymptotic to the rays $\Gamma_{(k ; \pm \varepsilon / 2)}$.

In addition to the sets $\Omega_{+}$and $\Omega_{-}$, we need their intersection

$$
\Omega_{0}:=\Omega_{+} \cap \Omega_{-} .
$$

The set $\Omega_{0}$ is depicted in Figure 5 . Observe that the properties of the sets $\Omega_{+}$and $\Omega_{-}$ imply the following properties of the set $\Omega_{0}$ :

- $\Omega_{0}=\bigcup_{k=1}^{6} \omega_{k}, \quad \overline{\omega_{k}} \subset \Omega_{k}^{\rho} \backslash \Gamma_{k}^{\rho}$, and each subset $\omega_{k}$ is open and connected;

- $\partial \omega_{k}=\gamma_{k}^{+}+\gamma_{k}^{-}$;

- $\operatorname{dist}\left(\gamma_{k}^{+}, \gamma_{k}^{-}\right) \geq c_{\varepsilon}$. 


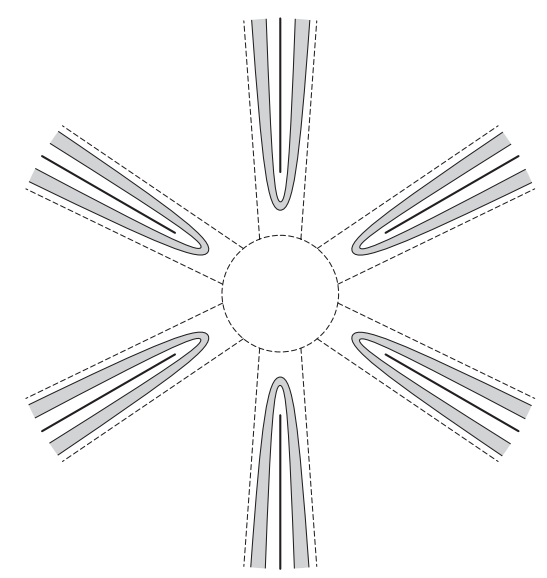

Figure 5. The set $\Omega_{0}=\Omega_{-} \cap \Omega_{+}=\bigcup_{k=1}^{6} \omega_{k}$.

Now, we are ready to introduce the principal ingredient of our proof, i.e., the model functions $g_{k}(\lambda, x)$ mentioned above. Assuming that $\lambda \in \Omega_{k}^{\rho} \backslash \Gamma_{k}^{\rho}$, we put

$$
\begin{aligned}
g_{k}(\lambda, x)= & \exp \left\{\frac{1}{2 \pi i} \int_{\Gamma_{k}^{\rho}} \frac{\ln G_{k}(\mu, x)}{\mu-\lambda} d \mu\right\} \\
& =\left\{\begin{array}{lll}
\left(\begin{array}{cc}
1 & \frac{s_{k}}{2 \pi i} \int_{\Gamma_{k}^{\rho}} \frac{e^{-2 \theta(\mu, x)}}{\mu-\lambda} d \mu \\
0 & 1
\end{array}\right), \quad k=2 l, \\
\left(\begin{array}{cc}
1 & 0 \\
\frac{s_{k}}{2 \pi i} \int_{\Gamma_{k}^{\rho}} \frac{e^{2 \theta(\mu, x)}}{\mu-\lambda} d \mu & 1
\end{array}\right), & k=2 l-1,
\end{array}\right.
\end{aligned}
$$

where the jump matrices $G_{k}(\lambda, x)$ and the phase function $\theta(\lambda, x)$ are given in (46). The characteristic property of $g_{k}(\lambda, x)$ is the jump relation, namely,

$$
g_{k+}(\lambda, x)=g_{k-}(\lambda, x) G_{k}(\lambda, x), \quad \lambda \in \Gamma_{k}^{\rho} \cap\{|\lambda| \geq 2 \rho+\delta\},
$$

where $g_{k+}(\lambda, x)$ and $g_{k-}(\lambda, x)$ denote the boundary values of $g_{k}(\lambda, x)$ as $\lambda$ approaches $\Gamma_{k}^{\rho}$ from the left and from the right, respectively.

On the set $\Omega_{0} \times \mathcal{K}$, the function $g(\lambda, x)$ can be defined by setting

$$
\left.g(\lambda, x)\right|_{\omega_{k}}=g_{k}(\lambda, x) .
$$

We list some direct consequences of the explicit formulas (48) and (50) and the geometric properties of the set $\Omega_{0}$ indicated above.

(i) $g(\lambda, x)$ is holomorphic both in $\lambda$ and in $x$. Indeed,

$$
g(\lambda, x) \in H^{0}\left(\Omega_{0} \times \mathcal{K}\right) .
$$

(ii) We have the uniform estimate

$$
\sup _{\lambda \in \Omega_{o}, x \in \mathcal{K}}|\lambda(g(\lambda, x)-I)| \leq c_{\rho, \mathcal{K}} .
$$

Moreover,

$$
c_{\rho, \mathcal{K}} \rightarrow 0 \quad \text { as } \rho \rightarrow \infty .
$$

The next lemma is a local version of the Birkhoff-Grothendieck theorem with parameter. This is the main technical step in our proof of Sibuya's Theorem $4^{\prime}$. The basic idea is the uniform boundedness of the relevant Cauchy operators (see Proposition 1 below), 


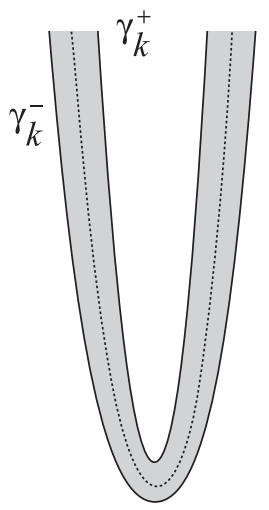

FIGURE 6. $\omega_{k}=\omega_{k}^{+} \cup \omega_{k}^{-}$.

which is the main advantage of moving to the horseshoe domains $\omega_{k}$ from the rays $\Gamma_{k}^{\rho}$. First, this idea was suggested in [1] (see also [7] and Appendix 1).

Lemma 2 (The basic factorization lemma). For sufficiently large $\rho>0$, there exist functions $\Psi_{ \pm}(\lambda, x)$ with the following properties.

$$
\begin{aligned}
& \text { 1. } \Psi_{ \pm}(\lambda, x) \in H^{0}\left(\Omega_{ \pm} \times \mathcal{K}\right) . \\
& \text { 2. } \sup _{(\lambda, x) \in \Omega_{ \pm} \times \mathcal{K}}\left|\lambda\left(I-\Psi_{ \pm}(\lambda, x)\right)\right| \leq C . \\
& \text { 3. } \Psi_{+}(\lambda, x) g(\lambda, x)=\Psi_{-}(\lambda, x), \quad \lambda \in \Omega_{0}, \quad x \in \mathcal{K} .
\end{aligned}
$$

Proof. We introduce three other Banach spaces:

$$
\begin{aligned}
H & =\left\{f \in H\left(\Omega_{0} \times \mathcal{K}\right), \sup _{(\lambda, x) \in \Omega_{0} \times \mathcal{K}}|\lambda f(\lambda, x)|<\infty\right\}, \\
H_{ \pm} & =\left\{f \in H\left(\Omega_{ \pm} \times \mathcal{K}\right), \sup _{(\lambda, x) \in \Omega_{ \pm} \times \mathcal{K}}|\lambda f(\lambda, x)|<\infty\right\} \subset H, \\
\|f\|_{H} & \equiv \sup _{(\lambda, x) \in \Omega_{0} \times \mathcal{K}}|\lambda f(\lambda, x)|, \quad\|f\|_{H_{ \pm}} \equiv \sup _{(\lambda, x) \in \Omega_{ \pm} \times \mathcal{K}}|\lambda f(\lambda, x)| .
\end{aligned}
$$

Given $f \in H$, we can define the Cauchy operators

$$
\left(P_{ \pm} f\right)(\lambda, x)=\frac{1}{2 \pi i} \int_{\gamma^{ \pm}} \frac{f(\mu, x)}{\mu-\lambda} d \mu, \quad \lambda \in \Omega_{ \pm} .
$$

We recall that $\gamma^{ \pm}=\partial \Omega_{ \pm}$, and that

$$
\partial \Omega_{0}=\gamma^{+}+\gamma^{-}=\sum_{k=1}^{6} \partial \omega_{k}=\sum_{k=1}^{6}\left(\gamma_{k}^{+}+\gamma_{k}^{-}\right) .
$$

Note that the Cauchy formula

$$
f(\lambda, x)=\left(P_{+} f\right)(\lambda, x)+\left(P_{-} f\right)(\lambda, x), \quad \lambda \in \Omega_{0},
$$

is valid for the functions in $H$.

Proposition 1. $P_{ \pm}$are bounded operators from $H$ to $H_{ \pm}$.

Proof of the proposition (an adaptation of the technique of [1]). Each $\omega_{k}$ can be represented as the union of the domains $\omega_{k}^{+}$and $\omega_{k}^{-}$in accordance with Figure 6. Assume that $\lambda \in \Omega_{+} \backslash \bigcup_{k} \omega_{k}^{+}$. 
Then

$$
\begin{aligned}
\lambda\left(P_{+} f\right)(\lambda, x) & =\frac{1}{2 \pi i} \int_{\gamma^{+}} \frac{\lambda f(\mu, x)}{\mu-\lambda} d \mu \\
\left|\lambda\left(P_{+} f\right)(\lambda, x)\right| & \leq \frac{1}{2 \pi} \int_{\gamma^{+}}|f(\mu, x)|^{2}|d \mu| \cdot|\lambda| \cdot \int_{\gamma^{+}} \frac{|d \mu|}{|\mu-\lambda|^{2}} \\
& \leq\|f\|_{H} \cdot \frac{1}{2 \pi} \int_{\gamma^{+}} \frac{|d \mu|}{|\mu|^{2}} \cdot \int_{\gamma^{+}} \frac{|d \mu| /|\lambda|}{\left(1+\left|\frac{\mu}{\lambda}\right|^{2}-2\left|\frac{\mu}{\lambda}\right| \cos \frac{\varepsilon}{12}\right)} \\
& \leq\|f\|_{H} \cdot \frac{1}{2 \pi} \int_{\gamma^{+}} \frac{|d \mu|}{|\mu|^{2}} \cdot 2 \int_{0}^{\infty} \frac{d z}{1+z^{2}-2 z \cos \frac{\varepsilon}{12}} \equiv C_{+} \cdot\|f\|_{H} .
\end{aligned}
$$

Similarly, if $\lambda \in \Omega_{-} \backslash \bigcup_{k} \omega_{k}^{-}$, then

$$
\left|\lambda\left(P_{-} f\right)(\lambda, x)\right| \leq C_{-} \cdot\|f\|_{H} .
$$

Suppose now that $\lambda \in \omega_{k}^{+}$. Then, we can use identity (53) and rewrite $\lambda\left(P_{+} f\right)(\lambda, x)$ as

$$
\lambda\left(P_{+} f\right)(\lambda, x)=\lambda f(\lambda, x)+\lambda\left(P_{-} f\right)(\lambda, x) .
$$

On the other hand, $\lambda \in \omega_{k}^{+} \Rightarrow \lambda \in \Omega_{-} \backslash \bigcup_{k} \omega_{k}^{-}$, and hence we can use (154) on the righthand side of (55)). Therefore (cf. [1] and the proof of Lemma A.1 in Appendix 1), we obtain

$$
\left|\lambda\left(P_{+} f\right)(\lambda, x)\right| \leq\left(1+C_{-}\right) \cdot\|f\|_{H}
$$

if $\lambda \in \omega_{k}^{+}$. In other words, we have the inequality

$$
\left|\lambda\left(P_{+} f\right)(\lambda, x)\right| \leq C \cdot\|f\|_{H} \quad \text { with } C:=\max \left\{C_{+}, 1+C_{-}\right\},
$$

for all $(\lambda, x) \in \overline{\Omega_{+}} \times \mathcal{K}$, which implies

$$
\left\|P_{+} f\right\|_{H_{+}} \leq C \cdot\|f\|_{H} .
$$

Similarly,

$$
\left\|P_{-} f\right\|_{H_{-}} \leq C^{\prime} \cdot\|f\|_{H}
$$

with

$$
C^{\prime}:=\max \left\{C_{-}, 1+C_{+}\right\}
$$

and the proposition is proved.

Put

$$
g(\lambda, x)=I+\stackrel{o}{g}(\lambda, x), \quad \stackrel{o}{g}(\lambda, x) \in H .
$$

We are looking for

$$
\Psi_{ \pm}(\lambda, x)=I+\stackrel{o}{\Psi}_{ \pm}(\lambda, x), \quad \stackrel{o}{\Psi}_{ \pm}(\lambda, x) \in H_{ \pm},
$$

such that

$$
I+\stackrel{o}{\Psi}_{-}=\left(I+\stackrel{o}{\Psi}_{+}\right)(I+\stackrel{o}{g}),
$$

or

$$
\stackrel{o}{\Psi}-\stackrel{o}{\Psi}+\stackrel{o}{\Psi}_{+} \stackrel{o}{g}+\stackrel{o}{g}
$$

Applying $P_{+}$, we see that

$$
0=\stackrel{o}{\Psi}_{+}+P_{+}(\stackrel{o}{\Psi} \stackrel{o}{g})+P_{+}(\stackrel{o}{g}) .
$$

Hence, we must only solve the following integral equation:

$$
\stackrel{o}{\Psi}++P_{+}[\stackrel{o}{\Psi} \stackrel{o}{g}]=-P_{+} \stackrel{o}{g} .
$$


Because of estimates (51) and (52), we conclude that the operator

$$
I+P_{+}[\stackrel{\circ}{g}]
$$

is invertible in the Banach space $H_{+}$for sufficiently large $\rho$; moreover, the norm of $\Psi^{o}+$ can be made sufficiently small to ensure the invertibility of the matrix $\Psi_{+}(\lambda, x)$ for all $(\lambda, x) \in \overline{\Omega_{+}} \times \mathcal{K}$. This completes the proof of the lemma.

Proof of Theorem $4^{\prime}$. Define the functions $Y_{k}(\lambda, x)$ as indicated in Figure 7 . We claim that these functions satisfy all the properties announced in the theorem.

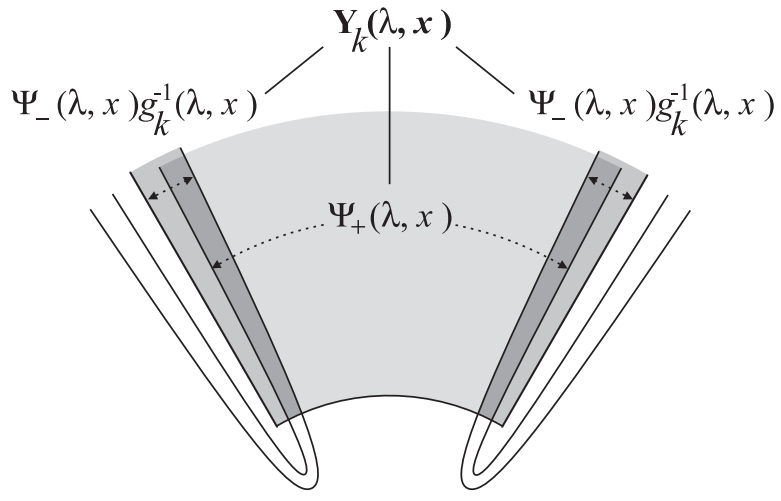

Figure 7 . The functions $Y_{k}(\lambda, x)$.

The properties that do not follow directly from the definition of the functions $Y_{k}(\lambda, x)$ and, therefore, need a proof, are the asymptotic statements $3-5$. The proof is based on the use of appropriate integral representations for the functions $Y_{k}(\lambda, x)$, which can be obtained as follows (cf. [30]).

First, we note that property 2 of the functions $\Psi_{ \pm}(\lambda, x)$ implies the estimate

$$
\sup _{(\lambda, x) \in \mathcal{L}_{k} \times \mathcal{K}}\left|\lambda\left(I-Y_{k}(\lambda, x)\right)\right| \leq C,
$$

which allows us to use the Cauchy formula in the sectors $\mathcal{L}_{k}$ and obtain the equations

$$
Y_{k}(\lambda, x)=I+\int_{\partial \mathcal{L}_{k}} \frac{Y_{k}(\mu, x)-I}{\mu-\lambda} \frac{d \mu}{2 \pi i}, \quad \lambda \in \stackrel{o}{\mathcal{L}}_{k},
$$

and

$$
0=\int_{\partial \mathcal{L}_{k}} \frac{Y_{k}(\mu, x)-I}{\mu-\lambda} \frac{d \mu}{2 \pi i}, \quad \lambda \notin \mathcal{L}_{k}
$$

where $\stackrel{o}{\mathcal{L}}_{k}$ denotes the interior of $\mathcal{L}_{k}$. We put

$$
\Gamma_{k}:=\Gamma_{k}^{\rho} \cap\{|\lambda| \geq 3 \rho\},
$$

and let $C_{k}$ be the arc of the circle $|\lambda|=3 \rho$ between the rays $\Gamma_{k-1}$ and $\Gamma_{k}$ (see Figure 2). Assume that $C_{k}$ is oriented counterclockwise. Then

$$
\partial \mathcal{L}_{k}=-\Gamma_{k}-C_{k}+\Gamma_{k-1},
$$


and we can rewrite (57) as

$$
\begin{aligned}
Y_{k}(\lambda, x)= & I-\int_{C_{k}} \frac{Y_{k}(\mu, x)-I}{\mu-\lambda} \frac{d \mu}{2 \pi i} \\
& -\int_{\Gamma_{k}} \frac{Y_{k}(\mu, x)-I}{\mu-\lambda} \frac{d \mu}{2 \pi i}+\int_{\Gamma_{k-1}} \frac{Y_{k}(\mu, x)-I}{\mu-\lambda} \frac{d \mu}{2 \pi i},
\end{aligned}
$$

where we keep $\lambda \in \stackrel{o}{\mathcal{L}}_{k}$. Observe that from property 2 of $Y_{k}$ we have

$$
Y_{k}(\lambda, x)-I=-Y_{k}(\lambda, x)\left(G_{k}(\lambda, x)-I\right)+Y_{k+1}(\lambda, x)-I, \quad \lambda \in \Gamma_{k},
$$

and

$$
Y_{k}(\lambda, x)-I=Y_{k-1}(\lambda, x)\left(G_{k-1}(\lambda, x)-I\right)+Y_{k-1}(\lambda, x)-I, \quad \lambda \in \Gamma_{k-1} .
$$

Therefore, 59] can be transformed into

$$
\begin{aligned}
Y_{k}(\lambda, x)= & I-\int_{C_{k}} \frac{Y_{k}(\mu, x)-I}{\mu-\lambda} \frac{d \mu}{2 \pi i} \\
& +\int_{\Gamma_{k}} \frac{Y_{k}(\mu, x)\left(G_{k}(\mu, x)-I\right)}{\mu-\lambda} \frac{d \mu}{2 \pi i}+\int_{\Gamma_{k-1}} \frac{Y_{k-1}(\mu, x)\left(G_{k-1}(\mu, x)-I\right)}{\mu-\lambda} \frac{d \mu}{2 \pi i} \\
& -\int_{\Gamma_{k}} \frac{Y_{k+1}(\mu, x)-I}{\mu-\lambda} \frac{d \mu}{2 \pi i}+\int_{\Gamma_{k-1}} \frac{Y_{k-1}(\mu, x)-I}{\mu-\lambda} \frac{d \mu}{2 \pi i} .
\end{aligned}
$$

Now, making use of the second Cauchy relation (58), we can replace the last two integrals by

and

$$
\int_{C_{k+1}} \frac{Y_{k+1}(\mu, x)-I}{\mu-\lambda} \frac{d \mu}{2 \pi i}+\int_{\Gamma_{k+1}} \frac{Y_{k+1}(\mu, x)-I}{\mu-\lambda} \frac{d \mu}{2 \pi i}
$$

$$
-\int_{C_{k-1}} \frac{Y_{k-1}(\mu, x)-I}{\mu-\lambda} \frac{d \mu}{2 \pi i}+\int_{\Gamma_{k-2}} \frac{Y_{k-1}(\mu, x)-I}{\mu-\lambda} \frac{d \mu}{2 \pi i},
$$

respectively. Thus, equation (60) can further be transformed into the equation $(61)$

$$
\begin{aligned}
Y_{k}(\lambda, x)= & I-\int_{C_{k+1}} \frac{Y_{k+1}(\mu, x)-I}{\mu-\lambda} \frac{d \mu}{2 \pi i}-\int_{C_{k}} \frac{Y_{k}(\mu, x)-I}{\mu-\lambda} \frac{d \mu}{2 \pi i} \\
& -\int_{C_{k-1}} \frac{Y_{k-1}(\mu, x)-I}{\mu-\lambda} \frac{d \mu}{2 \pi i} \\
& +\int_{\Gamma_{k}} \frac{Y_{k}(\mu, x)\left(G_{k}(\mu, x)-I\right)}{\mu-\lambda} \frac{d \mu}{2 \pi i}+\int_{\Gamma_{k-1}} \frac{Y_{k-1}(\mu, x)\left(G_{k-1}(\mu, x)-I\right)}{\mu-\lambda} \frac{d \mu}{2 \pi i} \\
& -\int_{\Gamma_{k+1}} \frac{Y_{k+1}(\mu, x)-I}{\mu-\lambda} \frac{d \mu}{2 \pi i}+\int_{\Gamma_{k-2}} \frac{Y_{k-1}(\mu, x)-I}{\mu-\lambda} \frac{d \mu}{2 \pi i} .
\end{aligned}
$$

Comparing the two integrals in the last row of (61) with similar integrals in (59), we see that the manipulations that led us from (59) to (61) result in the increase of the index $k$ in the first integral and its decrease in the second one. Therefore, repeating the above procedure several times, we shall arrive at the following final integral representation for $Y_{k}(\lambda, x)$ :

$$
Y_{k}(\lambda, x)=I-\sum_{l=1}^{6} \int_{C_{l}} \frac{Y_{l}(\mu, x)-I}{\mu-\lambda} \frac{d \mu}{2 \pi i}+\sum_{j=1}^{6} \int_{\Gamma_{l}} \frac{Y_{l}(\mu, x)\left(G_{l}(\mu, x)-I\right)}{\mu-\lambda} \frac{d \mu}{2 \pi i}
$$

for all $\lambda \in \stackrel{o}{\mathcal{L}}_{k}$. 
Each term of the first sum on the right in (62) is holomorphic in a neighborhood of $\lambda=\infty$, while the integrand of each term in the second sum decays exponentially as $\lambda \rightarrow \infty$. In fact, there exist positive constants $C$ and $c$ such that

$$
\left|Y_{l}(\mu, x)\left(G_{l}(\mu, x)-I\right)\right|<C e^{-c|\mu|^{3}}, \quad \mu \in \Gamma_{l}, \quad x \in \mathcal{K} .
$$

This means that, indeed, uniformly in $x \in \mathcal{K}$, we have the full asymptotic series (47) for $Y_{k}(\lambda, x)$ with coefficients given by the equations

$$
\begin{aligned}
m_{j}^{0}(x)= & \sum_{l=1}^{6} \int_{C_{l}}\left(Y_{l}(\mu, x)-I\right) \mu^{j-1} \frac{d \mu}{2 \pi i} \\
& -\sum_{l=1}^{6} \int_{\Gamma_{l}} Y_{l}(\mu, x)\left(G_{l}(\mu, x)-I\right) \mu^{j-1} \frac{d \mu}{2 \pi i} .
\end{aligned}
$$

In particular, from this equations it follows that all $m_{j}^{0}(x)$ are holomorphic functions.

By standard arguments, the integral representation (62) implies the $x$ - and $\lambda$-differentiability of the asymptotic expression (47). In fact, the $\lambda$ and $x$-differentiability of (47) is a direct consequence of its uniformity (see, e.g., [33]).

The last thing we have to comment on is that the asymptotic expansion (47) is valid in the closed sector $\mathcal{L}_{k}$ (in fact, in some sector that includes $\mathcal{L}_{k}$ ). Indeed, because of property 2, each of the functions $Y_{k}(\lambda, x)$ can be continued analytically beyond the boundaries of $\mathcal{L}_{k}$ so that it become holomorphic and satisfy the estimate (56) in a sector that includes $\mathcal{L}_{k}$. Therefore, in the representation (62) we can slightly rotate the rays $\Gamma_{k}$ and $\Gamma_{k-1}$ so as to make the representation (consequently, the asymptotic expansion (47)) valid for all $\lambda \in \mathcal{L}_{k}$.

4.2. The local solution of the Riemann-Hilbert problem. We denote by $\Omega_{0}, \Omega_{\infty}$, and $\omega$ the following subsets of the Riemann sphere $\mathbb{C} P^{1}$ :

$$
\begin{aligned}
\Omega_{0} & =\{\lambda \in \mathbb{C}:|\lambda| \leq R\}, \\
\Omega_{\infty} & =\{\lambda \in \mathbb{C} \cup \infty:|\lambda| \geq 4 \rho\}, \\
\omega & =\Omega_{0} \cap \Omega_{\infty},
\end{aligned}
$$

where $R>4 \rho$. Also, we denote by $D_{\delta}\left(x_{0}\right)$ the closed disk in the $x$-plane of radius $\delta$ and centered at $x_{0}$, i.e.,

$$
D_{\delta}\left(x_{0}\right)=\left\{x \in \mathbb{C}:\left|x-x_{0}\right| \leq \delta\right\} .
$$

Let $M(\lambda, x)$ be the Sibuya function discussed in the preceding subsection and corresponding to the choice

$$
\mathcal{K}=D_{\delta}\left(x_{0}\right)
$$

Observe that

$$
M(\lambda, x) \in H^{0}\left(\left(\Omega_{\infty} \backslash\{\infty\}\right) \times D_{\delta}\left(x_{0}\right)\right)
$$

for all $\rho \geq \rho_{0}\left(D_{\delta}\left(x_{0}\right)\right) \equiv \rho_{0}\left(x_{0}, \delta\right)$. In particular,

$$
M(\lambda, x) \in H^{0}\left(\omega \times D_{\delta}\left(x_{0}\right)\right) .
$$

By direct application of the Birkhoff-Grothendieck theorem with parameter (see Appendix 1) to the function $M(\lambda, x)$, we arrive at the following result.

Theorem 5. Let $x_{0} \in \mathbb{C}$. Then there exists a number $\delta_{0}>0$, a finite set $\Theta_{0} \equiv\left\{x_{0 j}\right\} \subset$ $D_{\delta_{0}}\left(x_{0}\right)$, and matrix-valued functions $T(\lambda, x)$ and $\Phi(\lambda, x)$ with the following properties.

- $T(\lambda, x)$ and $T^{-1}(\lambda, x)$ are holomorphic in $\Omega_{\infty} \times\left(D_{\delta_{0}}\left(x_{0}\right) \backslash \Theta_{0}\right)$ and meromorphic along $\Omega_{\infty} \times \Theta_{0}$ (i.e., they have poles at $x_{0 j} \in \Theta_{0}$, and the coefficients of the corresponding Laurent series are holomorphic in $\left.\Omega_{\infty}\right)$. 
- $\Phi(\lambda, x)$ and $\Phi^{-1}(\lambda, x)$ are holomorphic in $\Omega_{0} \times\left(D_{\delta_{0}}\left(x_{0}\right) \backslash \Theta_{0}\right)$ and meromorphic along $\Omega_{0} \times \Theta_{0}$.

$$
M(\lambda, x)=T^{-1}(\lambda, x) \lambda\left(\begin{array}{cc}
\kappa_{1} & 0 \\
0 & \kappa_{2}
\end{array}\right) \Phi(\lambda, x),
$$

where $\kappa_{1} \geq \kappa_{2}$ are integers, the same for all $x \in D_{\delta_{0}}\left(x_{0}\right) \backslash \Theta_{0}$.

The specific features of the function $M(\lambda, x)$ we are dealing with yield specifications of some of the statements above.

Proposition 2. The function $\Phi(\lambda, x)$ is an entire function of $\lambda$; indeed,

$$
\Phi(\lambda, x) \in H^{0}\left(\mathbb{C} \times\left(D_{\delta_{0}}\left(x_{0}\right) \backslash \Theta_{0}\right)\right),
$$

and both $\Phi(\lambda, x)$ and $\Phi^{-1}(\lambda, x)$ are meromorphic along $D_{0} \times \Theta_{0}$. Moreover,

$$
\begin{array}{r}
\Phi(\lambda, x) S_{1} \cdots S_{k-1} \simeq \lambda\left(\begin{array}{cc}
-\kappa_{1} & 0 \\
0 & -\kappa_{2}
\end{array}\right) B_{0}\left(I+\sum_{j=1}^{\infty} \frac{m_{j}(x)}{\lambda^{j}}\right) e^{-\theta(\lambda, x) \sigma_{3}}, \\
\lambda \rightarrow \infty, \quad \lambda \in \mathcal{L}_{k}, \quad \operatorname{det} B_{0} \neq 0,
\end{array}
$$

where the coefficients $m_{j}(x)$ are holomorphic in $D_{\delta_{0}}\left(x_{0}\right) \backslash \Theta_{0}$ and meromorphic in $D_{\delta_{0}}\left(x_{0}\right)$. The asymptotics is uniform in $x \in D$, where $D$ is an arbitrary compact subset of $D_{\delta_{0}}\left(x_{0}\right) \backslash \Theta_{0}$, and it is differentiable with respect to both $x$ and $\lambda$.

The proposition is an immediate consequence of equations (64), (66), and the asymptotic properties of $M(\lambda, x)$ (see (45)). We note also that the coefficients $m_{j}(x)$ are finite combinations of the coefficients $m_{j}^{0}(x)$ and the coefficients of the Taylor expansions of $T(\lambda, x)$ at $\lambda=\infty$.

Proposition 3. The numbers $\kappa_{1}$ and $\kappa_{2}$ in (66) satisfy the equation

$$
\kappa_{1}+\kappa_{2}=0 .
$$

Proof. The matrix equation (66) implies the following relation in the annulus $\omega=\Omega_{0} \cap$ $\Omega_{\infty}$ for the corresponding determinants:

$$
\operatorname{det} \Phi(\lambda, x)=\operatorname{det} T(\lambda, x) \operatorname{det} M(\lambda, x) \lambda^{-\kappa_{1}-\kappa_{2}} .
$$

Simultaneously, we observe that the asymptotic properties of the matrix-valued function $M(\lambda, x)$ (see statement 2 of Theorem 4) yield

$$
\operatorname{det} M \rightarrow 1 \quad \text { as } \lambda \rightarrow \infty \text {. }
$$

Suppose that $\kappa_{1}+\kappa_{2} \neq 0$ and put

$$
\phi(\lambda, x):= \begin{cases}\operatorname{det} \Phi(\lambda, x) & \text { if } \lambda \in \Omega_{0}, \\ \operatorname{det} T(\lambda, x) \operatorname{det} M(\lambda, x) \lambda^{-\kappa_{1}-\kappa_{2}} & \text { if } \lambda \in \Omega_{\infty} \backslash\{\infty\}\end{cases}
$$

$\left(x \in D_{\delta_{0}}\left(x_{0}\right) \backslash \Theta_{0}\right)$. Equations (69) and (70) mean that, for every $x$, the (entire) function $\phi_{x}(\lambda) \equiv \phi(\lambda, x)$ is either identically zero $\left(\kappa_{1}+\kappa_{2}>0\right)$ or is a polynomial of degree $-\kappa_{1}-\kappa_{2}\left(\kappa_{1}+\kappa_{2}<0\right)$. In both cases there are points $\lambda$ on the finite complex plane where the matrix $\Phi(\lambda, x)$ is not invertible. This contradicts Proposition 2; hence, the sum $\kappa_{1}+\kappa_{2}$ must be zero.

Equation (68) allows us to specify (66) as follows:

$$
M(\lambda, x)=T^{-1}(\lambda, x) \lambda^{\kappa \sigma_{3}} \Phi(\lambda, x),
$$

where $\kappa \geq 0$ is an integer (the same for all $x \in D_{\delta_{0}}\left(x_{0}\right) \backslash \Theta_{0}$ ); we recall that

$$
\sigma_{3}=\left(\begin{array}{cc}
1 & 0 \\
0 & -1
\end{array}\right) \text {. }
$$


Proposition 4 (cf. [20]). The number $\kappa$ in equation (71) is (identically) zero.

Proof. It is in this statement that we use the assumption (44). Suppose that $\kappa>0$. Note that then the functions $T(\lambda, x)$ and $\Phi(\lambda, x)$ in (66) are defined up to the following transformations:

$$
\begin{aligned}
& T(\lambda, x) \mapsto \tilde{T}(\lambda, x)=\left(\begin{array}{cc}
c_{1} & 0 \\
\sum_{j=0}^{2 \kappa} a_{j} \lambda^{j-2 \kappa} & c_{2}
\end{array}\right) T(\lambda, x), \\
& \Phi(\lambda, x) \mapsto \tilde{\Phi}(\lambda, x)=\left(\begin{array}{cc}
c_{1} & 0 \\
\sum_{j=0}^{2 \kappa} a_{j} \lambda^{j} & c_{2}
\end{array}\right) \Phi(\lambda, x),
\end{aligned}
$$

where $c_{1} c_{2} \neq 0$ and $a_{0}, \ldots, a_{2 \kappa}$ are arbitrary. Using this freedom, one can adjust the matrix-valued functions $T(\lambda, x)$ and $\Phi(\lambda, x)$ in such a way (perhaps adding a few more points to the singular set $\Theta_{0}$ and reducing the radius $\delta_{0}$ a little bit) that asymptotic relation (67) can be transformed to the relation

$$
\begin{array}{r}
\Phi(\lambda, x) S_{1} \cdots S_{k-1} \simeq\left(I+\sum_{j=1}^{\infty} \frac{m_{j}(x)}{\lambda^{j}}\right) e^{-(\theta(\lambda, x)+\kappa \ln \lambda) \sigma_{3}}, \\
\lambda \rightarrow \infty, \quad \lambda \in \mathcal{L}_{k},
\end{array}
$$

where the coefficients $m_{k}(x)$ are meromorphic in $D_{\delta_{0}}\left(x_{0}\right)$ and holomorphic in $D_{\delta_{0}}\left(x_{0}\right) \backslash \Theta_{0}$ and satisfy

$$
\left(m_{j}\right)_{12} \equiv 0, \quad j<2 \kappa .
$$

It should also be noted that the asymptotic expression (72) is still uniform and differentiable with respect to both $x$ and $\lambda$.

When applied to the logarithmic derivatives $\Phi_{\lambda} \Phi^{-1}$ and $\Phi_{x} \Phi^{-1}$, the arguments based on the Liouville theorem and used already in $\S 3$ in the proof of Theorem 2 show that the function $\Phi(\lambda, x)$ satisfies the isomonodromy Lax pair (31). The functional parameters $u(x), v(x), w(x)$, and $y(x)$ are meromorphic in $D_{\delta_{0}}\left(x_{0}\right)$ and holomorphic in $D_{\delta_{0}}\left(x_{0}\right) \backslash \Theta_{0}$, and they satisfy the general relations

$$
\begin{aligned}
& w=u_{x}, \quad y=v_{x}, \\
& \nu+\kappa=v w-u y .
\end{aligned}
$$

Moreover,

$$
u=2\left(m_{1}\right)_{12} \quad \text { and } \quad v=2\left(m_{1}\right)_{21} .
$$

From the first equation and (73) we conclude that

$$
u(x) \equiv 0 .
$$

By (74), this means that $w(x) \equiv 0$ as well, and therefore the right-hand side of (75) is identically zero; but it is not zero, since $\nu$ is not an integer. This contradiction proves that $\kappa$ must be zero 9

Propositions 2-4 yield the following corollary of Theorem 5, which constitutes the local solution (with respect to $x$ ) of the original $\mathrm{RH}$ problem.

Theorem 6. Let $x_{0} \in \mathbb{C}$. Then there exist a number $\delta_{0}>0$, a finite set $\Theta_{0} \equiv\left\{x_{0 j}\right\} \subset$ $D_{\delta_{0}}\left(x_{0}\right)$, and a unique matrix-valued function $\Psi_{0}(\lambda, x)$ with the following properties.

\footnotetext{
${ }^{9}$ Strictly speaking, in the proof we have excluded the case where $T_{11}(\infty, x) \equiv 0$. If $T_{11}(\infty, x) \equiv 0$, then $\kappa$ must be replaced by $-\kappa$ in (72), and "12" by "21" in (73). The rest of the proof will go along the same lines as before.
} 
(i) $\Psi_{0}(\lambda, x)$ and $\Psi_{0}^{-1}(\lambda, x)$ are holomorphic in $\mathbb{C} \times\left(D_{\delta_{0}}\left(x_{0}\right) \backslash \Theta_{0}\right)$ and meromorphic along $\mathbb{C} \times \Theta_{0}$.

(ii) $\operatorname{det} \Psi_{0}(\lambda, x) \equiv 1$.

(iii) For every $x \in D_{\delta_{0}}\left(x_{0}\right) \backslash \Theta_{0}$ we have

$$
\begin{array}{r}
\Psi_{0}(\lambda, x) S_{1} \cdots S_{k-1} \simeq\left(I+\sum_{j=1}^{\infty} \frac{m_{j}(x)}{\lambda^{j}}\right) e^{-\theta(\lambda, x) \sigma_{3}}, \\
\lambda \rightarrow \infty, \quad \lambda \in \mathcal{L}_{k},
\end{array}
$$

where the coefficients $m_{j}(x)$ are holomorphic in $D_{\delta_{0}}\left(x_{0}\right) \backslash \Theta_{0}$ and meromorphic in $D_{\delta_{0}}\left(x_{0}\right)$. The asymptotic expression is uniform in $x \in D_{\delta_{0}}\left(x_{0}\right) \backslash \Theta_{0}^{\varepsilon}$ and is differentiable with respect to both $x$ and $\lambda . \Theta_{0}^{\varepsilon}$ denotes an arbitrary $\varepsilon$-neighborhood of the set $\Theta_{0}$.

Proof. Let $\Phi(\lambda, x)$ and $T(\lambda, x)$ be the functions mentioned in Theorem 5. Set

$$
\Psi_{0}(\lambda, x):=T^{-1}(\infty, x) \Phi(\lambda, x) .
$$

We argue that this function satisfies conditions (i)-(iii) above. Indeed, condition (i) follows directly from Proposition 2 and the analytic properties of $T(\lambda, x)$. By (71) and Proposition 4, the equation

$$
\Psi_{0}(\lambda, x)=T^{-1}(\infty, x) T(\lambda, x) M(\lambda, x)
$$

is fulfilled. Note that

$$
T^{-1}(\infty, x) T(\lambda, x)=I+\sum_{j=1}^{\infty} T_{j}(x) \lambda^{-j},
$$

where the series converges in $\Omega_{\infty}$ uniformly for $x \in D, \bar{D} \subset D_{\delta_{0}}\left(x_{0}\right) \backslash \Theta_{0}$, and the coefficients $T_{j}(x)$ are holomorphic in $D_{\delta_{0}}\left(x_{0}\right) \backslash \Theta_{0}$ and meromorphic in $D_{\delta_{0}}\left(x_{0}\right)$. Hence, taking into account the asymptotic properties of the Sibuya function $M(\lambda, x)$ (see statement 2 of Theorem 4), we arrive at the asymptotic relation (iii). To obtain (ii), we note that for every fixed $x$ we have

$$
\operatorname{det} \Psi_{0}(\lambda, x) \rightarrow 1, \quad \lambda \rightarrow \infty,
$$

and (ii) follows via the Liouville theorem. Finally, to prove the uniqueness statement of the theorem, we assume that $\tilde{\Psi}_{0}(\lambda, x)$ is another function satisfying (i)-(iii) and consider the ratio

$$
R(\lambda, x):=\tilde{\Psi}_{0}(\lambda, x) \Psi_{0}^{-1}(\lambda, x) .
$$

For every fixed $x, R(\lambda, x)$ is an entire function of $\lambda$. Moreover, as $\lambda \rightarrow \infty$ and $\lambda \in \mathcal{L}_{k}$, by (iii) we obtain

$$
\begin{aligned}
R(\lambda, x) & =\tilde{\Psi}_{0}(\lambda, x) \Psi_{0}^{-1}(\lambda, x) \\
& =\left[\tilde{\Psi}_{0}(\lambda, x) S_{1} \cdots S_{k-1} e^{\theta(\lambda, x) \sigma_{3}}\right]\left[\Psi_{0}(\lambda, x) S_{1} \cdots S_{k-1} e^{\theta(\lambda, x) \sigma_{3}}\right]^{-1} \\
& =I+\mathcal{O}(1 / \lambda) .
\end{aligned}
$$

In other words, in the entire neighborhood of infinity we have $R(\lambda, x) \rightarrow I, \quad \lambda \rightarrow \infty$, whence $R(\lambda, x) \equiv I$. Uniqueness and, with it, the theorem are proved. 
4.3. The solution of the Riemann-Hilbert problem. By Theorem 6 and countable compactness of the complex plane, there exists a countable covering

$$
\mathbb{C}=\bigcup_{n=1}^{\infty} D_{\delta_{n}}\left(x_{n}\right)
$$

of the complex $x$-plane by disks $D_{\delta_{n}}\left(x_{n}\right)$ such that for each $D_{\delta_{n}}\left(x_{n}\right)$ the statement of Theorem 6 is valid. In other words, for every $n$ there exists a finite set $\Theta_{n} \equiv\left\{x_{n j}\right\} \subset$ $D_{\delta_{n}}\left(x_{n}\right)$ and a unique matrix-valued function $\Psi_{n}(\lambda, x)$ such that:

(i) $\Psi_{n}(\lambda, x)$ and $\Psi_{n}^{-1}(\lambda, x)$ are holomorphic in $\mathbb{C} \times\left(D_{\delta_{n}}\left(x_{n}\right) \backslash \Theta_{n}\right)$ and meromorphic along $\mathbb{C} \times \Theta_{n}$

(ii) $\operatorname{det} \Psi_{n}(\lambda, x) \equiv 1$;

(iii) for every $x \in D_{\delta_{n}}\left(x_{n}\right) \backslash \Theta_{n}$ we have

$$
\begin{array}{r}
\Psi_{n}(\lambda, x) S_{1} \cdots S_{k-1} \simeq\left(I+\sum_{j=1}^{\infty} \frac{m_{j}(x)}{\lambda^{j}}\right) e^{-\theta(\lambda, x) \sigma_{3}}, \\
\lambda \rightarrow \infty, \quad \lambda \in \mathcal{L}_{k},
\end{array}
$$

where the coefficients $m_{j}(x)$ are holomorphic in $D_{\delta_{n}}\left(x_{n}\right) \backslash \Theta_{n}$ and meromorphic in $D_{\delta_{n}}\left(x_{n}\right)$; the asymptotic expression is uniform in $x \in D$, where $D$ is an arbitrary compact subset of $D_{\delta_{n}}\left(x_{n}\right) \backslash \Theta_{n}$, and it is differentiable with respect to both $x$ and $\lambda$.

Now our aim is to glue this collection of solutions (local in $x$ ) of the original RiemannHilbert problem into a global function $\Psi(\lambda, x)$, which should solve the problem for all complex $x \in \mathbb{C} \backslash \Theta$, where

$$
\Theta:=\bigcup_{n=1}^{\infty} \Theta_{n} .
$$

(Note that the set $\Theta$ is countable and has $\infty$ as its only limit point.) The following lemma plays a central role in the construction.

Lemma 3. Suppose that

$$
\stackrel{\circ}{D} \delta_{n}\left(x_{n}\right) \cap \stackrel{\circ}{D} \delta_{m}\left(x_{m}\right) \neq \varnothing
$$

Then

$$
\Psi_{n}(\lambda, x)=\Psi_{m}(\lambda, x), \quad(\lambda, x) \in \mathbb{C} \times\left(\left(D_{\delta_{n}}\left(x_{n}\right) \backslash \Theta_{n}\right) \cap\left(D_{\delta_{m}}\left(x_{m}\right) \backslash \Theta_{m}\right)\right) .
$$

Proof. The proof is literally the same as that of the uniqueness statement in Theorem 6 . The principal point is that the two functions have one and the same asymptotic behavior (with exactly the same Stokes matrices) as $\lambda \rightarrow \infty$.

Now, we put

$$
\Psi(\lambda, x):=\Psi_{n}(\lambda, x), \quad(\lambda, x) \in \mathbb{C} \times\left(D_{\delta_{n}}\left(x_{n}\right) \backslash \Theta_{n}\right) .
$$

Because of Lemma 3 , this equation determines $\Psi(\lambda, x)$ as a holomorphically invertible function on the entire product $\mathbb{C} \times(\mathbb{C} \backslash \Theta)$. Moreover, it inherits all the common analytic properties of the local solutions $\Psi_{n}(\lambda, x)$, including uniqueness. In other words, the function $\Psi(\lambda, x)$ satisfies all the properties announced in Theorem 1.

Remark 3 . The poles of the functions $u(x ; s)$ and $v(x ; s)$ are the values of the parameter $x$ for which the generalized inverse monodromy problem with the given monodromy data $s=\left\{S_{1}, \ldots, S_{6} ; \nu\right\}$ is not solvable. At the same time, by using the Schlesinger transformations indicated in Lemma 1 , we can see that if $x$ is a pole of the functions 
$u(x ; s)$ and $v(x ; s)$, then the generalized monodromy problem is solvable for each of the following choices of the monodromy data:

$$
\hat{s}=\left\{S_{1}, \ldots, S_{6} ; \nu-1\right\}
$$

and

$$
\tilde{s}=\left\{S_{1}, \ldots, S_{6} ; \nu+1\right\} .
$$

Remark 4. The Sibuya function $M(\lambda, x)$ determines a holomorphic vector bundle over $\mathbb{C} P^{1}$. The points $x_{j}$ of the set $\Theta \equiv \Theta(s)$, i.e., the poles of the functions $u(x ; s)$ and $v(x ; s)$, are the points where the bundle is not trivial. If this is the case, the index $\kappa$ in the representation (71) is equal to 1 .

For more details concerning the facts mentioned in Remarks 3 and 4, see [20].

\section{Appendix 1. The Birkhoff-Grothendieck theorem With a PARAmeter}

Denote by $\Omega_{0}, \Omega_{\infty}$, and $\omega$ the following subsets of the Riemann sphere $\mathbb{C} P^{1}$ :

$$
\begin{aligned}
\Omega_{0} & =\{\lambda \in \mathbb{C}:|\lambda| \leq R\}, \\
\Omega_{\infty} & =\{\lambda \in \mathbb{C} \cup \infty:|\lambda| \geq r\}, \\
\omega & =\Omega_{0} \cap \Omega_{\infty},
\end{aligned}
$$

where $R>r$. Also, as in the main text, we denote by $D_{\delta}\left(x_{0}\right)$ the closed disk in the $x$-plane of radius $\delta$ and centered at $x_{0}$, i.e.,

$$
D_{\delta}\left(x_{0}\right)=\left\{x \in \mathbb{C}:\left|x-x_{0}\right| \leq \delta\right\} .
$$

Our goal is to present an elementary proof of the following (local) version of the classical Birkhoff-Grothendieck theorem with a parameter.

Theorem A.1. Suppose $M(\lambda, x)$ belongs to the space $H^{0}\left(\omega \times D_{\delta}\left(x_{0}\right)\right)$. Then there exists a number $0<\delta_{0} \leq \delta$, a finite set $\Theta_{0} \equiv\left\{x_{0 j}\right\} \subset D_{\delta_{0}}\left(x_{0}\right)$, and matrix-valued functions $T(\lambda, x)$ and $\Phi(\lambda, x)$ such that the following statements are true.

- The functions $T(\lambda, x)$ and $T^{-1}(\lambda, x)$ are holomorphic in $\Omega_{\infty} \times\left(D_{\delta_{0}}\left(x_{0}\right) \backslash \Theta_{0}\right)$ and meromorphic along $\Omega_{\infty} \times \Theta_{0}$ (i.e., they have poles at $x_{0 j} \in \Theta_{0}$, and the coefficients of the corresponding Laurent series are holomorphic in $\left.\Omega_{\infty}\right)$.

- $\Phi(\lambda, x)$ and $\Phi^{-1}(\lambda, x)$ are holomorphic in $\Omega_{0} \times\left(D_{\delta_{0}}\left(x_{0}\right) \backslash \Theta_{0}\right)$ and meromorphic along $\Omega_{0} \times \Theta_{0}$.

- We have

$$
M(\lambda, x)=T^{-1}(\lambda, x) \lambda\left(\begin{array}{cc}
\kappa_{1} & 0 \\
0 & \kappa_{2}
\end{array}\right) \Phi(\lambda, x),
$$

where $\kappa_{1} \geq \kappa_{2}$ are some integers, the same for all $x \in D_{\delta_{0}}\left(x_{0}\right) \backslash \Theta_{0}$.

We shall prove the theorem in a series of lemmas following closely the proof in [7, where the case of the general matrix dimension and the presence of an arbitrary finite number of parameters was considered. As in [7], the first two lemmas are merely $2 \times 2$ specifications and modifications (for the case of the presence of the parameter $x$ ) of the corresponding statements proved in [1. Lemma A.3 is a particular case of the Sauvage lemma (see [17]). Also, as it has already been indicated in the Introduction, Theorem A.1 itself is a particular case of the general theorem of Malgrange (see [27]).

The Banach spaces $H\left(\omega \times D_{\delta}\left(x_{0}\right)\right), H\left(\Omega_{\infty} \times D_{\delta}\left(x_{0}\right)\right)$, and $H\left(\Omega_{0} \times D_{\delta}\left(x_{0}\right)\right)$ are equipped with the standard norm $\|f\|=\max _{(\lambda, x)}|f(\lambda, x)|$. In addition to the subspaces $H^{0}\left(\Omega_{\infty, 0} \times D_{\delta}\left(x_{0}\right)\right)$, we introduce yet another subspace, $\hat{H}\left(\Omega_{\infty} \times D_{\delta}\left(x_{0}\right)\right)$, of $H\left(\Omega_{\infty} \times D_{\delta}\left(x_{0}\right)\right)$ consisting of all $f(\lambda, x) \in H\left(\Omega_{\infty} \times D_{\delta}\left(x_{0}\right)\right)$ such that $f(\infty, x) \equiv 0$. We also note that, obviously, the spaces $H\left(\Omega_{\infty, 0} \times D_{\delta}\left(x_{0}\right)\right)$ are subspaces of $H\left(\omega \times D_{\delta}\left(x_{0}\right)\right)$. 
Lemma A.1. Under the conditions of the theorem, if $\|M-I\|<\varepsilon$ for sufficiently small $\varepsilon>0$, then there exist unique functions $T(\lambda, x) \in H^{0}\left(\Omega_{\infty} \times D_{\delta}\left(x_{0}\right)\right)$ and $\Phi(\lambda, x) \in$ $H^{0}\left(\Omega_{0} \times D_{\delta}\left(x_{0}\right)\right)$ such that $T(\infty, x) \equiv I$ and

$$
M(\lambda, x)=T^{-1}(\lambda, x) \Phi(\lambda, x) .
$$

Proof. Much as in the proof of the basic factorization lemma in $\S 4$ (and, once again, following [1]), we introduce the Cauchy operators $P_{ \pm}: H\left(\omega \times D_{\delta}\left(x_{0}\right)\right) \mapsto H\left(\Omega_{0, \infty} \times\right.$ $\left.D_{\delta}\left(x_{0}\right)\right)$ by the equations

$$
\left(P_{+} f\right)(\lambda, x)=\frac{1}{2 \pi i} \oint_{|\mu|=R} \frac{f(\mu, x)}{\mu-\lambda} d \mu, \quad\left(P_{-} f\right)(\lambda, x)=-\frac{1}{2 \pi i} \oint_{|\mu|=r} \frac{f(\mu, x)}{\mu-\lambda} d \mu,
$$

where the orientation in both integrals is counterclockwise. We show that, like the operators in $\S 4$, the operators $P_{ \pm}$are bounded. Once again the crucial role is played by the Cauchy formula

$$
f(\lambda, x)=\left(P_{+} f\right)(\lambda, x)+\left(P_{-} f\right)(\lambda, x),
$$

which is true for any $f \in H\left(\omega \times D_{\delta}\left(x_{0}\right)\right)$. Note also that

$$
\operatorname{Im} P_{-}=\hat{H}\left(\Omega_{\infty} \times D_{\delta}\left(x_{0}\right)\right) .
$$

We denote by $s$ the number $(R-r) / 2$. If $0 \leq|\lambda|<s+r$, then

$$
\left|\left(P_{+} f\right)(\lambda, x)\right| \leq \frac{1}{2 \pi} \oint_{|\mu|=R}\left|\frac{f(\mu, x)}{\mu-\lambda}\right||d \mu| \leq\|f\| \frac{1}{2 \pi} \oint_{|\mu|=R} \frac{1}{s}|d \mu| \leq \frac{R}{s}\|f\| .
$$

If $s+r \leq|\lambda| \leq R$, we use (799) and obtain

$$
\begin{aligned}
& \left|\left(P_{+} f\right)(\lambda, x)\right| \leq\|f\|+\left|\left(P_{-} f\right)(\lambda, x)\right| \\
& \quad \leq\|f\|+\frac{1}{2 \pi} \oint_{|\mu|=r}\left|\frac{f(\mu, x)}{\mu-\lambda}\right||d \mu| \leq\|f\|+\|f\| \frac{1}{2 \pi} \oint_{|\mu|=r} \frac{1}{s}|d \mu| \leq \frac{s+r}{s}\|f\| .
\end{aligned}
$$

Thus,

$$
\left|\left(P_{+} f\right)(\lambda, x)\right| \leq \max \left\{\frac{R}{s}, \frac{s+r}{s}\right\}\|f\|
$$

for all $(\lambda, x) \in \Omega_{0} \times D_{\delta}\left(x_{0}\right)$, whence

$$
\left\|P_{+}\right\| \leq C:=\max \left\{\frac{R}{s}, \frac{s+r}{s}\right\} .
$$

Similar arguments show that the same estimate is true also for the norm of the operator $P_{-}$.

Put $N(\lambda, x):=M(\lambda, x)-I$; then $\|N\|<\varepsilon$. To prove the existence statement of the lemma, it suffices to show the existence of a matrix-valued function $X(\lambda, x) \in \hat{H}\left(\Omega_{\infty} \times\right.$ $\left.D_{\delta}\left(x_{0}\right)\right)$ such that $\|X\|<1 / 2$ and

$$
P_{-}((I+X) M)=0 .
$$

Indeed, in this case we can put

$$
T(\lambda, x):=I+X(\lambda, x) \in H^{0}\left(\Omega_{\infty} \times D_{\delta}\left(x_{0}\right)\right)
$$

and

$$
\Phi(\lambda, x):=T(\lambda, x) M(\lambda, x) \in H^{0}\left(\Omega_{0} \times D_{\delta}\left(x_{0}\right)\right) .
$$

By (179) and (80), it is clear that the function $\Phi(\lambda, x)$ is in $H\left(\Omega_{0} \times D_{\delta}\left(x_{0}\right)\right)$, and we only need to comment on the holomorphic invertibility of $\Phi(\lambda, x)$. We have

$$
\Phi=I+X+(I+X) N
$$


or (by application of the identity $\Phi=P_{+}(\Phi)$ )

$$
\Phi=I+P_{+}((I+X) N)
$$

and the norm $\left\|P_{+}((I+X) N)<\right\| P_{+} \| \frac{3 \varepsilon}{2}$ can be made smaller than $1 / 2$ for sufficiently small $\varepsilon$.

Equation 80 can be rewritten as follows:

$$
\begin{aligned}
& P_{-}((I+X) M)=P_{-}((I+X)(I+N)) \\
& \quad=P_{-}((I+X+X N+N))=X+P_{-}(X N)+P_{-}(N)=0
\end{aligned}
$$

where we have used the obvious identities

$$
P_{-}(X)=X \quad \text { and } \quad P_{-}(I)=0 .
$$

Therefore, the equation we need to solve can be written as the integral equation

$$
X+P_{-}(X N)=-P_{-}(N)
$$

in the space $\hat{H}\left(\Omega_{\infty} \times D_{\delta}\left(x_{0}\right)\right)$. Since the operator $P_{-}$is bounded and $\|N\|<\varepsilon$, for sufficiently small $\varepsilon$ the operator $I+P_{-}[\cdot N]$ is invertible, and the norm of the solution $X=\left(I+P_{-}[\cdot N]\right)^{-1}\left(-P_{-}(N)\right)$ can be made as small as we need. This proves the existence part of the lemma.

Suppose $\tilde{T}(\lambda, x)$ and $\tilde{\Phi}(\lambda, x)$ form another pair of functions with the same properties. Then $M=T^{-1} \Phi=\tilde{T}^{-1} \tilde{\Phi}$, and the function

$$
R(\lambda, x):= \begin{cases}\tilde{T}(\lambda, x) T^{-1}(\lambda, x) & \text { if } \lambda \in \Omega_{\infty}, \\ \tilde{\Phi}(\lambda, x) \Phi^{-1}(\lambda, x) & \text { if } \lambda \in \Omega_{0}\end{cases}
$$

is a holomorphically invertible matrix-valued function in $\overline{\mathbb{C}} \times D_{\delta}\left(x_{0}\right)$. Thus, by the Liouville theorem, $R(\lambda, x)$ does not depend on $\lambda$. On the other hand, from the construction we see that $R(\infty, x) \equiv I$, so that $R(\lambda, x) \equiv I$ and, hence, $\tilde{T}(\lambda, x) \equiv T(\lambda, x)$, $\tilde{\Phi}(\lambda, x) \equiv \Phi(\lambda, x)$.

The second step of the proof of the theorem is the replacement of the function $M(\lambda, x)$ by a certain matrix-valued function rational in $\lambda$.

Lemma A.2. For every matrix-valued function $M(\lambda, x) \in H^{0}\left(\omega \times D_{\delta}\left(x_{0}\right)\right)$, there exists a number $0<\delta^{\prime \prime} \leq \delta$, a matrix-valued function $F(\lambda, x)$ rational in $\lambda$ with coefficients holomorphic in $x$, and matrix-valued functions $T(\lambda, x), \Phi(\lambda, x)$ such that the following statements are true.

1. The only possible poles of $F(\lambda, x)$ are $\lambda=0$ and $\lambda=\infty$. Moreover,

$$
\alpha_{0, \infty}:=\sup _{x \in D_{\delta}\left(x_{0}\right)}(\text { the order of the pole at } 0, \infty)<\infty .
$$

2. $F(\lambda, x)$ is holomorphically invertible in $\omega \times D_{\delta^{\prime \prime}}\left(x_{0}\right)$, and the positions of the zeros of $\operatorname{det} F(\lambda, x)$ in $\Omega_{0} \backslash \omega$ do not depend on $x$.

3. $T(\lambda, x)$ and $T^{-1}(\lambda, x)$ are holomorphic in $\Omega_{\infty} \times D_{\delta^{\prime \prime}}\left(x_{0}\right)$.

4. $\Phi(\lambda, x)$ and $\Phi^{-1}(\lambda, x)$ are holomorphic in $\Omega_{0} \times D_{\delta^{\prime \prime}}\left(x_{0}\right)$.

5. $M(\lambda, x)=T^{-1}(\lambda, x) F(\lambda, x) \Phi(\lambda, x)$.

Proof. The function $M\left(\lambda, x_{0}\right)$ can be uniformly approximated in $\omega$ by a matrix-valued function rational in $\lambda$ with possible poles at 0 and $\infty$ only. For this, we can merely use parts of the Laurent expansion of $M\left(\lambda, x_{0}\right)$ in the annulus $\omega$, cutting the infinite tails of the expansion in an appropriate way.

On the other hand, for every $\varepsilon>0$ we can choose a number $\delta^{\prime}>0$ such that $\left|M^{-1}(\lambda, x) M\left(\lambda, x_{0}\right)-I\right|<\varepsilon$ in $\omega \times D_{\delta^{\prime}}\left(x_{0}\right)$. 
Thus, we can always find a number $0<\delta^{\prime} \leq \delta$ and a matrix-valued function $B(\lambda)$ holomorphically invertible in $\omega$, rational in $\lambda$, with poles at 0 and $\infty$ only, and such that $\left|B^{-1}(\lambda) M(\lambda, x)-I\right|$ is uniformly small in $\omega \times D_{\delta^{\prime}}\left(x_{0}\right)$. Applying Lemma A.1 to the product $B^{-1}(\lambda) M(\lambda, x)$, we obtain

$$
B^{-1} M=T_{1}^{-1} \Phi_{1}, \quad M=B T_{1}^{-1} \Phi_{1},
$$

where $T_{1}(\lambda, x) \in H^{0}\left(\Omega_{\infty} \times D_{\delta^{\prime}}\left(x_{0}\right)\right), \Phi_{1}(\lambda, x) \in H^{0}\left(\Omega_{0} \times D_{\delta^{\prime}}\left(x_{0}\right)\right)$. Consider the function $B(\lambda) T_{1}^{-1}(\lambda, x)$ and approximate it from the right in a similar way by a function $H(\lambda)$ with the same properties as $B$. Applying Lemma A.1 once again, we get

$$
B T_{1}^{-1} H^{-1}=T_{2}^{-1} \Phi_{2}
$$

where $T_{2}(\lambda, x), \Phi_{2}(\lambda, x)$ have the same properties as $T_{1}(\lambda, x), \Phi_{1}(\lambda, x)$ (for some $0<$ $\left.\delta^{\prime \prime} \leq \delta^{\prime}\right)$. Consequently,

$$
\Phi_{2}=T_{2} B T_{1}^{-1} H^{-1}, \quad M=T_{2}^{-1} \Phi_{2} H \Phi_{1}=T_{2}^{-1} F \Phi_{1}, \quad F:=\Phi_{2} H .
$$

From the first relation above it follows that for every $x \in D_{\delta^{\prime \prime}}\left(x_{0}\right)$ the matrix-valued function $\Phi_{2}(\lambda, x)$ is meromorphic in $\Omega_{\infty}$ with poles independent of $x$ and with Laurent series coefficients holomorphic in $x$. On the other hand, $\Phi_{2}$ is holomorphically invertible in $\Omega_{0} \times D_{\delta^{\prime \prime}}\left(x_{0}\right)$ by construction. Thus, $\Phi_{2}$ is rational in $\lambda$ with coefficients holomorphic in $x$. In its turn, this implies exactly the same properties for $F$. Moreover, $F(\lambda, x)$ is holomorphically invertible in $\omega \times D_{\delta^{\prime \prime}}\left(x_{0}\right)$, because so are $\Phi_{2}(\lambda, x)$ and $H(\lambda)$.

Finally, we see that

$$
F= \begin{cases}T_{2} B T_{1}^{-1} & \text { if } \lambda \in \Omega_{\infty} \\ \Phi_{2} H & \text { if } \lambda \in \Omega_{0}\end{cases}
$$

Therefore, the only possible poles of $F$ are 0 and $\infty$. Moreover, the corresponding maximal orders $\alpha_{0}$ and $\alpha_{\infty}$ of the poles do not exceed the orders of the corresponding poles of the matrix-valued functions $H(\lambda)$ and $B(\lambda)$, respectively (these functions are independent of $x$ ). Also, the position of the zeros of $\operatorname{det} F(\lambda, x)$ in $\left(\Omega_{0} \backslash \omega\right) \times D_{\delta^{\prime \prime}}\left(x_{0}\right)$ does not depend on $x$ because $\operatorname{det} \Phi_{2}$ has no zeros in $\Omega_{0}$ and $H$ does not depend on $x$ at all.

Now, denoting $T=T_{2}$ and $\Phi=\Phi_{1}$, we get the decomposition (5).

The following lemma is a crucial step in the proof of the theorem. In fact, it proves the theorem in the particular case of rational matrices $M$, without any small norm assumptions.

It is convenient to introduce more notation. Let $\Omega$ be a subset of the extended complex $\lambda$-plane, and let $\Theta$ be a finite subset of the interior of the disk $D_{\delta}\left(x_{0}\right)$ on the complex $x$-plane. We denote by $H^{0}\left(\Omega \times\left(D_{\delta}\left(x_{0}\right) \backslash \Theta\right)\right)$ the space of matrix-valued functions $f(\lambda, x)$ such that $f(\lambda, x)$ and $f^{-1}(\lambda, x)$ are holomorphic in $\Omega \times\left(D_{\delta}\left(x_{0}\right) \backslash \Theta\right)$ and meromorphic along $\Omega \times \Theta$.

Lemma A.3. Let $M(\lambda, x) \in H^{0}\left(\left(\Omega_{0} \backslash\{0\}\right) \times\left(D_{\delta}\left(x_{0}\right) \backslash \Theta\right)\right)$, where $\Theta$ is a finite subset of the interior of $D_{\delta}\left(x_{0}\right)$. Moreover, we assume that $M(\lambda, x)$ is rational in $\lambda$ with the coefficients meromorphic in $D_{\delta}\left(x_{0}\right)$ and holomorphic in $D_{\delta}\left(x_{0}\right) \backslash \Theta$. Suppose also that the only possible poles of $M$ (as a function of $\lambda$ ) are $\lambda=0$ and $\lambda=\infty$, and that the corresponding maximal orders $\alpha_{0}$ and $\alpha_{\infty}$ of the poles are finite. Then there exists a number $\delta_{0} \leq \delta$, a finite set $\Theta_{0} \subset D_{\delta_{0}}\left(x_{0}\right), \Theta \subset \Theta_{0}$, and matrix-valued functions $T(\lambda, x)$ and $\Phi(\lambda, x)$ such that the following statements are true.

- $T(\lambda, x)$ and $T^{-1}(\lambda, x)$ are holomorphic in $(\overline{\mathbb{C}} \backslash\{0\}) \times\left(D_{\delta_{0}}\left(x_{0}\right) \backslash \Theta_{0}\right)$ and meromorphic along $\overline{\mathbb{C}} \backslash\{0\} \times \Theta_{0}$. In other words,

$$
T(\lambda, x) \in H^{0}\left((\overline{\mathbb{C}} \backslash\{0\}) \times\left(D_{\delta_{0}}\left(x_{0}\right) \backslash \Theta_{0}\right)\right) .
$$

- $\Phi(\lambda, x)$ and $\Phi^{-1}(\lambda, x)$ are holomorphic in $\Omega_{0} \times\left(D_{\delta_{0}}\left(x_{0}\right) \backslash \Theta_{0}\right)$ and meromorphic along $\Omega_{0} \times \Theta_{0}$. In other words,

$$
\Phi(\lambda, x) \in H^{0}\left(\Omega_{0} \times\left(D_{\delta_{0}}\left(x_{0}\right) \backslash \Theta_{0}\right)\right) .
$$

Moreover, $\Phi(\lambda, x)$ is a polynomial in $\lambda$ with the coefficients meromorphic in $D_{\delta_{0}}\left(x_{0}\right)$ and holomorphic in $D_{\delta_{0}}\left(x_{0}\right) \backslash \Theta_{0}$, and also $\operatorname{deg} \Phi(\lambda, x) \leq \alpha_{0}+\alpha_{\infty}$ for 
- We have

$$
M(\lambda, x)=T^{-1}(\lambda, x) \lambda^{\left(\begin{array}{cc}
\kappa_{1} & 0 \\
0 & \kappa_{2}
\end{array}\right)} \Phi(\lambda, x),
$$

where $\kappa_{1} \geq \kappa_{2}$ are some integers that are the same for all $x \in D_{\delta_{0}}\left(x_{0}\right) \backslash \Theta_{0}$. 
Proof. Since the only poles of $M$ in the $\lambda$-plane are 0 and $\infty$, we may assume that the matrix-valued function $M$ is already of the form

$$
M(\lambda, x)=\lambda\left(\begin{array}{cc}
\kappa_{1} & 0 \\
0 & \kappa_{2}
\end{array}\right) L(\lambda, x)
$$

for some integers $\kappa_{1} \geq \kappa_{2}$ and a polynomial matrix $L(\lambda, x)$,

$$
L(\lambda, x)=L_{0}(x)+L_{1}(x) \lambda+\cdots+L_{m}(x) \lambda^{m} .
$$

(Take simply $\kappa_{1}=\kappa_{2}=-\alpha_{0}$.) We note that

$$
L(\lambda, x) \in H^{0}\left(\left(\Omega_{0} \backslash\{0\}\right) \times\left(D_{\delta}\left(x_{0}\right) \backslash \Theta\right)\right)
$$

and the degree $m$ of the polynomial $L(\lambda, x)$ can be assumed to be the same for all $x$ (for instance, equal to $\alpha_{0}+\alpha_{\infty}$, although for some $x$ the coefficient of the highest degree term may be zero). We also note that all the coefficients $L_{j}(x)$ are meromorphic in $D_{\delta}\left(x_{0}\right)$ with possible poles at the points of the set $\Theta$.

If for all $x \in D_{\delta}\left(x_{0}\right) \backslash \Theta$ we have $\operatorname{det} L(0, x) \neq 0$, then there is nothing to prove: simply put $\delta_{0} \equiv \delta, \Theta_{0} \equiv \Theta, T \equiv I$, and $\Phi \equiv L$.

Let $\operatorname{det} L\left(0, x_{1}\right)=0$ for some $x_{1} \in D_{\delta}\left(x_{0}\right) \backslash \Theta$. We claim that then

$$
\operatorname{det} L(0, x) \equiv 0 \text {. }
$$

To prove (84), first we observe that

$$
\operatorname{det} L(\lambda, x)=l_{0}(x)+l_{1}(x) \lambda+\cdots+l_{n}(x) \lambda^{n},
$$

for some integer $n$ (which we can choose the same for all $x$ ). All the coefficients $l_{k}(x)$ are meromorphic functions of $x \in D_{\delta}\left(x_{0}\right)$ with possible poles belonging to the set $\Theta$. By our assumption, $l_{0}\left(x_{1}\right)=0$. At the same time, for at least one $0<k \leq n$ we must have $l_{k}\left(x_{1}\right) \neq 0$; otherwise, $\operatorname{det} L\left(\lambda, x_{1}\right) \equiv \operatorname{det} L\left(0, x_{1}\right)=0$ in contradiction with (83). We denote by $k_{0}$ the maximal of such $k$ 's, and set

$$
P_{0}(\lambda, x):=l_{0}(x)+l_{1}(x) \lambda+\cdots+l_{k_{0}}(x) \lambda^{k_{0}} .
$$

Take $R_{0}<R$ so that the circle $C_{0}:=\left\{\lambda:|\lambda|=R_{0}\right\}$ does not pass through the zeros of $P_{0}\left(\lambda, x_{1}\right)$. By the choice of $k_{0}$, we have $\operatorname{det} L\left(\lambda, x_{1}\right)=P_{0}\left(\lambda, x_{1}\right)$. Therefore,

$$
\operatorname{det} L(\lambda, x)-P_{0}(\lambda, x) \rightarrow 0, \quad x \rightarrow x_{1},
$$

uniformly with respect to $\lambda \in C_{0}$. Simultaneously,

$$
P_{0}(\lambda, x) \rightarrow P_{0}\left(\lambda, x_{1}\right), \quad x \rightarrow x_{1},
$$

uniformly with respect to $\lambda \in C_{0}$. By the choice of $R_{0}$, we have $\left|P_{0}\left(\lambda, x_{1}\right)\right| \geq c_{0}>0$ for all $\lambda \in C_{0}$. Consequently, there exists $\delta^{\prime}>0$ such that

$$
\left|\operatorname{det} L(\lambda, x)-P_{0}(\lambda, x)\right|<\left|P_{0}(\lambda, x)\right|
$$

for all $\lambda \in C_{0}$ and all $x \in D_{\delta^{\prime}}\left(x_{1}\right) \subset D_{\delta}\left(x_{0}\right) \backslash \Theta$.

Now, suppose that (84) is not true. Then we can find $0<\delta^{\prime \prime} \leq \delta^{\prime}$ such that

$$
l_{0}(x) \neq 0, \quad x \in D_{\delta^{\prime \prime}}\left(x_{1}\right) \backslash\left\{x_{1}\right\} .
$$

Let $\lambda_{j}(x), j=1, \ldots, k_{0}$, be the zeros of the polynomial $P_{x}(\lambda):=P_{0}(\lambda, x)$. They satisfy the relation

$$
\prod_{j} \lambda_{j}(x)=\frac{l_{0}(x)}{l_{k_{0}}(x)}(-1)^{k_{0}} .
$$

Note that

$$
\left|l_{k_{0}}(x)\right| \geq c_{0}^{\prime}>0, \quad x \in D_{\delta^{\prime \prime \prime}}\left(x_{1}\right),
$$

for some $0<\delta^{\prime \prime \prime} \leq \delta^{\prime \prime}$. From this, the fact that $l_{0}(x) \rightarrow 0$ as $x \rightarrow x_{1}$, and equation (87), we conclude that in any neighborhood of $\lambda=0$ we can find at least one $\lambda_{j}(x)$ for some 
$x \in D_{\delta^{\prime \prime \prime}}\left(x_{1}\right) \backslash\left\{x_{1}\right\}$. In particular, for some $y \in D_{\delta^{\prime \prime \prime}}\left(x_{1}\right) \backslash\left\{x_{1}\right\}$ we have at least one zero of $P_{0}(\lambda, y)$ inside the circle $C_{0}$. By (85) and the Rouche theorem, inside the circle $C_{0}$ the polynomial $Q_{y}(\lambda):=\operatorname{det} L(\lambda, y)$ has at least one zero. Denote this zero by $\mu$. By (86), we have $Q_{y}(0) \neq 0$, and therefore $\mu \neq 0$. Thus, there is at least one pair $(\lambda, x)$ in $\left(\Omega_{0} \backslash\{0\}\right) \times D_{\delta}\left(x_{0}\right)$, namely $(\mu, y)$, for which $\operatorname{det} L(\lambda, x)=0$. This contradicts (83)), and hence proves (84).

We denote by $m_{1}(x)$ and $m_{2}(x)$ the rows of the matrix $L_{0}(x) \equiv L(0, x)$ and assume that

$$
m_{1}(x) \neq 0 \quad \text { for some } x .
$$

Then identity (84) implies the existence of a scalar function $s(x)$ rational in the entries of $L_{0}(x)$ and such that

$$
m_{2}(x)=s(x) m_{1}(x) .
$$

(For instance, we may take $s(x)=m_{21}(x) / m_{11}(x)$ if $m_{11}(x) \neq 0$ for some $x$.) Define

$$
T_{1}(\lambda, x):=\left(\begin{array}{cc}
1 & 0 \\
-s(x) \lambda^{k_{2}-k_{1}} & 1
\end{array}\right) .
$$

It is clear that $T_{1}(\lambda, x) \in H^{0}\left((\overline{\mathbb{C}} \backslash\{0\}) \times\left(D_{\delta}\left(x_{0}\right) \backslash \Theta_{1}\right)\right)$, where $\Theta_{1}$ is the set of poles of $s(x)$, i.e., the set $\Theta$ extended by the zeros of the relevant entry of $L_{0}(x)$. Restricting, if necessary, the $x$-domain to $D_{\delta_{1}}\left(x_{0}\right)$ with $\delta_{1}<\delta$, we can always make $\Theta_{1}$ finite (and still containing $\Theta$ ).

From (89) it follows immediately that

$$
T_{1}(\lambda, x) M(\lambda, x)=\lambda\left(\begin{array}{cc}
\kappa_{1} & 0 \\
0 & \kappa_{2}+1
\end{array}\right) L_{1}(\lambda, x),
$$

where

$$
L_{1}(\lambda, x)=\left(\begin{array}{cc}
1 & 0 \\
-s(x) \lambda^{-1} & \lambda^{-1}
\end{array}\right) L(\lambda, x) .
$$

Because of (89), the function $L_{1}(\lambda, x)$ is still a matrix polynomial in $\lambda$. Its coefficients are meromorphic in $D_{\delta_{1}}\left(x_{0}\right)$ and holomorphic in $D_{\delta_{1}}\left(x_{0}\right) \backslash \Theta_{1}$, and $\operatorname{deg} L_{1} \leq \operatorname{deg} L \leq \alpha_{0}+\alpha_{\infty}$. Also, equation (90) implies that $L_{1}$ inherits the basic analytic properties of $M$. Indeed,

$$
L_{1}(\lambda, x) \in H^{0}\left(\left(\Omega_{0} \backslash\{0\}\right) \times\left(D_{\delta_{1}}\left(x_{0}\right) \backslash \Theta_{1}\right)\right) .
$$

If (88) is not true, i.e., if $m_{1}(x) \equiv 0$, then we can increase the number $\kappa_{1}$ directly, without the procedure presented above. In fact, in this case we have

$$
M(\lambda, x)=\lambda\left(\begin{array}{cc}
\kappa_{1}+1 & 0 \\
0 & \kappa_{2}
\end{array}\right) L_{1}(\lambda, x),
$$

where

$$
L_{1}(\lambda, x)=\left(\begin{array}{cc}
\lambda^{-1} & 0 \\
0 & 1
\end{array}\right) L(\lambda, x)
$$

is again a polynomial satisfying (91).

Multiplying the right-hand side of (90) or (92) by a suitable constant matrix (either identity or $\sigma_{1}$ in fact), we can rewrite these equations in the form

$$
T^{\prime}(\lambda, x) M(\lambda, x)=\lambda^{\left(\begin{array}{cc}
\kappa_{1}^{\prime} & 0 \\
0 & \kappa_{2}^{\prime}
\end{array}\right)} L^{\prime}(\lambda, x),
$$

where $\kappa_{1}^{\prime} \geq \kappa_{2}^{\prime}$ and the matrices $T^{\prime}(\lambda, x)$ and $L^{\prime}(\lambda, x)$ have the same properties as the 
matrices $T_{1}(\lambda, x)$ and $L_{1}(\lambda, x)$, respectively. Since $\kappa_{1}^{\prime}+\kappa_{2}^{\prime}>\kappa_{1}+\kappa_{2}$, iterating (if necessary) the process presented above, in a finite number of step 19 we get $\operatorname{det} L^{\prime}(0, x) \neq$ 0 (for all $x$ ), and hence the decomposition (81) of the lemma. The final set $\Theta_{0}$ is obtained as the finite union of the $\Theta$-sets generated at every step of the procedure. This completes the proof of the lemma.

Now, we are in a position to prove the theorem.

Proof of the theorem. By Lemma A.2, we can restrict our consideration to the case of a matrix-valued function $M(\lambda, x)$ rational in $\lambda$ with coefficients holomorphic in $D_{\delta}\left(x_{0}\right)$ and such that the following is true.

1. The only possible poles of $M(\lambda, x)$ are $\lambda=0$ and $\lambda=\infty$. Moreover,

$$
\alpha_{0, \infty}:=\sup _{x \in D_{\delta}\left(x_{0}\right)}(\text { the order of the pole at } 0, \infty)<\infty
$$

2. The positions of the zeros of $\operatorname{det} M(\lambda, x)$ in $\Omega_{0} \backslash \omega$ do not depend on $x$.

If, moreover, $M(\lambda, x)$ is holomorphically invertible in $\left(\Omega_{0} \backslash\{0\}\right) \times D_{\delta}\left(x_{0}\right)$, then the theorem follows immediately from Lemma A.3 (with $\Theta=\varnothing$ ). Suppose that $\operatorname{det} M\left(\lambda_{0}, x\right)=0$ for some $0 \neq \lambda_{0} \in \Omega_{0} \backslash \omega$.

Consider the new local coordinates $\zeta=\lambda-\lambda_{0}$ and define the polynomial matrix-valued function $\tilde{M}(\zeta, x):=\left(\zeta+\lambda_{0}\right)^{\alpha_{0}} M\left(\zeta+\lambda_{0}\right)$. Let $\tilde{\Omega}_{0}$ denote a small disk in the $\zeta$-plane with center at $\zeta=0$ and such that the matrix-valued function $\tilde{M}(\zeta, x)$ is holomorphically invertible in $\left(\tilde{\Omega}_{0} \backslash\{0\}\right) \times D_{\delta}\left(x_{0}\right)$. Applying Lemma A.3 to $\tilde{M}(\zeta, x)$ (again starting with $\Theta=\varnothing)$, we obtain the representation

$$
M(\lambda, x)=T^{-1}(\lambda, x)\left(\lambda-\lambda_{0}\right)^{C} \Phi(\lambda, x),
$$

where $C=\operatorname{diag}\left(\kappa_{1}, \kappa_{2}\right), T(\lambda, x) \in H^{0}\left(\left(\overline{\mathbb{C}} \backslash\left\{\lambda_{0}\right\}\right) \times\left(D_{\delta_{0}}\left(x_{0}\right) \backslash \Theta_{0}\right)\right)$, and the matrix-valued function $\Phi(\lambda, x)$ is a polynomial in $\lambda$ whose coefficients are holomorphic in $D_{\delta_{0}}\left(x_{0}\right) \backslash \Theta_{0}$ and meromorphic in $D_{\delta_{0}}\left(x_{0}\right)$. Moreover, by construction, we have $\operatorname{det} \Phi\left(\lambda_{0}, x\right) \neq 0$, while from equation (93) it follows that $\Phi(\lambda, x)$ is holomorphically invertible in the annulus $\omega$ and its determinant has zeros in the set $\Omega_{0} \backslash\{0\}$ only at the possible zeros of $\operatorname{det} M$ different from $\lambda_{0}$. Noticing that the function $\left(\lambda /\left(\lambda-\lambda_{0}\right)\right)^{C}$ is holomorphically invertible in $\Omega_{\infty} \times\left(D_{\delta_{0}}\left(x_{0}\right) \backslash \Theta_{0}\right)$, from (93) we derive the equation

$$
M(\lambda, x)=T_{1}^{-1}(\lambda, x) M_{1}(\lambda, x),
$$

where $M_{1}(\lambda, x):=\lambda^{C} \Phi(\lambda, x)$ has all the properties of the original function $M(\lambda, x)$ except its determinant has one zero less in $\Omega_{0} \backslash \omega$. The function $T_{1}^{-1}(\lambda, x)$ has exactly the properties announced in the theorem. Repeating, if necessary, the above construction for the function $M_{1}(\lambda, x)$, after a finite number of steps we eliminate all the possible zeros of $\operatorname{det} M$ in $\Omega_{0} \backslash \omega$ and arrive at the function $M^{\prime}(\lambda, x)$ that satisfies the conditions of Lemma A.3 (with a nonempty singularity set $\Theta^{\prime}$, in general). Again, the theorem follows from the lemma.

\footnotetext{
${ }^{10}$ The process cannot be repeated infinitely many times because of the obvious relation det $M=$ $\pm \lambda^{\kappa_{1}+\kappa_{2}} \operatorname{det} L^{\prime}$ and the fact that, being a rational function, $\operatorname{det} M$ has a finite order of the pole at $\lambda=\infty$.
} 
Appendix 2. More on Painlevé equations

\section{Additional symmetries-particular case of P2.}

$$
\begin{aligned}
& \left\{\begin{array}{l}
u=v \\
w=y
\end{array} \Longleftrightarrow \sigma_{2} A(-\lambda) \sigma_{2}=A(\lambda)\right. \\
& \Longleftrightarrow \Psi_{k+3}\left(e^{i \pi} \lambda\right)=\sigma_{2} \Psi_{k}(\lambda) \sigma_{2} \\
& \Longleftrightarrow\left\{\begin{array}{l}
S_{k+3}=\sigma_{2} S_{k} \sigma_{2}, \\
\nu=0, \\
\sigma_{2}=\left(\begin{array}{cc}
0 & -i \\
i & 0
\end{array}\right), \\
s_{k+3}=-s_{k}, \\
s_{1}-s_{2}+s_{3}+s_{1} s_{2} s_{3}=0,
\end{array}\right. \\
& \left\{\begin{array}{l}
u=v \in \mathbb{R} \\
y=w \in \mathbb{R}
\end{array} \Longleftrightarrow \overline{A(\bar{\lambda})}=\sigma_{1} A(\lambda) \sigma_{1}\right. \\
& \Longleftrightarrow\left\{\begin{array}{c}
s_{k+3}=-s_{k} \\
s_{1}=\bar{s}_{3} \\
s_{2}=\bar{s}_{2}
\end{array}\right. \\
& \left\{\begin{array}{l}
u=v \in i \mathbb{R} \\
y=w \in i \mathbb{R}
\end{array} \Longleftrightarrow \overline{A(\bar{\lambda})}=\sigma_{2} A(\lambda) \sigma_{2}\right. \\
& \Longleftrightarrow\left\{\begin{array}{c}
s_{k+3}=-s_{k} \\
s_{1}=-\bar{s}_{3} \\
s_{2}=-\bar{s}_{2} .
\end{array}\right.
\end{aligned}
$$

General P2 and P34. The system

implies that

$$
\begin{aligned}
& u_{x x}=x u+2 u^{2} v, \\
& v_{x x}=x v+2 u v^{2}
\end{aligned}
$$

$$
u_{x} v-u v_{x}=\nu=\text { const }
$$

and the logarithmic derivatives

$$
p=\frac{u_{x}}{u}, \quad q=\frac{v_{x}}{v},
$$

solve P2 in a different scaling:

$$
\begin{aligned}
& p_{x x}=2 p^{3}-2 x p+1-2 \nu, \\
& q_{x x}=2 q^{3}-2 x q+1+2 \nu,
\end{aligned}
$$

while the product $w=u v$ solves P34:

$$
w_{x x}=\frac{w_{x}^{2}}{2 w}+4 w^{2}+2 x w-\frac{\nu^{2}}{2 w} .
$$

All the functions are related by the Bäcklund transformations:

$$
\begin{gathered}
w=\frac{1}{2}\left(p_{x}+p^{2}-x\right)=\frac{1}{2}\left(q_{x}+q^{2}-x\right), \\
w_{x}=2 p w-\nu=2 q w+\nu, \\
p=\frac{w_{x}+\nu}{2 w}, \quad p_{x}=x+2 w-p^{2}, \\
q=\frac{w_{x}-\nu}{2 w}, \quad q_{x}=x+2 w-q^{2},
\end{gathered}
$$




$$
\begin{gathered}
q=p-\frac{2 \nu}{p_{x}+p^{2}-x}, \quad p=q+\frac{2 \nu}{q_{x}+q^{2}-x}, \\
p_{x}+p^{2}=q_{x}+q^{2} .
\end{gathered}
$$

For more details, we refer the reader to [35].

\section{REFERENCES}

[1] D. V. Anosov and A. A. Bolibrukh, The Riemann-Hilbert problem, Aspects Math., vol. E 22, Friedr. Vieweg, Braunschweig, 1994. MR,1276272 (95d:32024)

[2] W. Balser, Meromorphic transformation to Birkhoff standard form in dimension three, J. Fac. Sci. Univ. Tokyo Sect. IA Math. 36 (1989), 233-246. MR1014998 (90m:34008)

[3] _ Formal power series and linear systems of meromorphic ordinary differential equations, Springer-Verlag, New York, 2000. MR1722871 (2001f:34169)

[4] A. A. Bolibrukh, The Riemann-Hilbert problem, Uspekhi Mat. Nauk 45 (1990), no. 2, 3-47; English transl., Russian Math. Surveys 45 (1990), no. 2, 1-58. MR.1069347 (92j:14014)

[5] — The Riemann-Hilbert problem on a complex projective straight line, Mat. Zametki 46 (1989), no. 3, 118-120. (Russian) MR1032917 (91e:14009)

[6] - On sufficient conditions for the existence of a Fuchsian equation with prescribed monodromy, J. Dynam. Control Systems 5 (1999), 453-472. MR1722011 (2000j:34117)

[7] _ Inverse problem for linear differential equations with meromorphic coefficients, Isomonodromic Deformations and Applications in Physics (Montréal, QC, 2000), CRM Proc. Lecture Notes, vol. 31, Amer. Math. Soc., Providence, RI, 2002, pp. 3-25. MR.1887344 (2003b:34171)

[8] R. Beals and D. H. Sattinger, Integrable systems and isomonodromy deformations, Phys. D 65 (1993), 17-47. MR 1221245 (94e:58051)

[9] O. Costin, Correlation between pole location and asymptotic behavior for Painlevé I solutions, MSRI Preprint no. 094-97, Math. Sci. Res. Inst., Berkeley, CA, 1997.

[10] P. A. Deift, UPenn lecture notes on the Riemann-Hilbert method, 2000.

[11] , Private communication (unpublished).

[12] P. A. Deift and X. Zhou, A steepest descent method for oscillatory Riemann-Hilbert problems. Asymptotics for the mkdv equation, Ann. of Math. (2) 137 (1993), 295-368. MR1207209 (94d:35143)

[13] _ Asymptotics for the Painlevé II equation, Comm. Pure Appl. Math. 48 (1995), 277-337. MR.1322812 (96d:34004)

[14] H. Flaschka and A. Newell, Monodromy- and spectrum-preserving deformations. I, Comm. Math. Phys. 76 (1980), 65-116. MR0588248 (82g:35103)

[15] A. S. Fokas and A. R. Its, The isomonodromy method and the Painlevé equations, Important Developments in Soliton Theory (A. S. Fokas, V. E. Zakharov, eds.), Springer, Berlin, 1993. MR1280472 (95j:34012)

[16] A. S. Fokas and X. Zhou, On the solvability of Painlevé II and IV, Comm. Math. Phys. 144 (1992), 601-622. MR:1158763 (93d:34004)

[17] P. Hartman, Ordinary differential equations, John Wiley and Sons, Inc., New York etc., 1964. MR.0171038(30:1270)

[18] A. R. Its, Connection formulae for the Painlevé transcendents, The Stokes Phenomenon and Hilbert's 16th Problem (Groningen, 1995) (B. L. J. Braaksma, G. K. Immink, and M. van der Put, eds.), World Sci. Publishing, River Edge, NJ, 1996, pp. 139-165. MR1443691 (98c:34008)

[19] A. R. Its and V. Yu. Novokshenov, The isomonodromic deformation method in the theory of Painlevé equations, Lecture Notes in Math., vol. 1191, Springer-Verlag, Berlin-New York, 1986. MR.0851569 (89b:34012)

[20] Effective sufficient conditions for the solvability of an inverse problem of monodromy theory for systems of linear ordinary differential equations, Funktsional. Anal. i Prilozhen. 22 (1988), no. 3, 25-36; English transl., Funct. Anal. Appl. 22 (1988), no. 3, 190-200. MR0961759 (90c:58167)

[21] A. R. Its, A. S. Fokas, and A. A. Kapaev, On the asymptotic analysis of the Painlevé equations via the isomonodromy method, Nonlinearity 7 (1994), 1291-1325. MR.1294544 (96c:34011)

[22] A. V. Kitaev, The justification of the asymptotic formulas obtained by the isomonodromic deformation method, Zap. Nauchn. Sem. Leningrad. Otdel. Mat. Inst. Steklov. (LOMI) 179 (1989), 101-109; English transl., J. Soviet. Math. 57 (1991), no. 3, 3131-3135. MR1039598 (91b:34087) 
[23] N. Joshi and M. D. Kruskal, An asymptotic approach to the connection problem for the first and the second Painlevé equations, Phys. Lett. A 130 (1988), 129-137; A simple proof that Painlevé equations have no movable essential singularities, Preprint no. CMA-R06-90, Centre for Math. Anal., Australian Nat. Univ., 1990. MR0948427 (90d:34016)

[24] M. Jimbo, T. Miwa, and K. Ueno, Monodromy preserving deformation of linear ordinary differential equations with rational coefficients. I, Phys. D 2 (1981), 306-352. MR.0630674(83k:34010a)

[25] W. B. Jurkat, D. A. Lutz, and A. Peyerimhoff, Birkhoff invariants and effective calculations for meromorphic linear differential equations. I, Math. Anal. Appl. 53 (1976), 438-470; II, Houston J. Math. 2 (1976), 207-238. MR0399544 (53:3388a) MR0399545 (53:3388b)

[26] V. P. Kostov, Fuchsian linear systems on $C P^{1}$ and the Riemann-Hilbert problem, C. R. Acad. Sci. Paris Ser. I Math. 315 (1992), no. 2, 143-148. MR.1197226 (94a:34007)

[27] B. Malgrange, La classification des connexions irrégulières à une variable, Mathematics and Physics (Paris, 1979/1982) (L. Boutet de Monvel, A. Douady, J.-L. Verdier, eds.), Progr. Math., vol. 37, Birkhäuser, Boston, MA, 1983, pp. 381-399; Sur les déformations isomonodromiques. I. Singularités régulières, ibid., pp. 401-426; Sur les déformations isomonodromiques. II. Singularités irrégulières, ibid., pp. 427-438. MR0728430 (85m:58006) MR0728431 (85m:58094a); MR0728432 (85m:58094b)

[28] L. J. Mason, M. A. Singer, and N. M. J. Woodhouse, Tau functions and the twistor theory of integrable systems, Preprint, Univ. of Oxford, 1999.

[29] T. Miwa, Painlevé property of monodromy preserving deformation equations and the analyticity of $\tau$-functions, Publ. Res. Inst. Math. Sci. 17 (1981), 703-712. MR0642657 (84k:34014)

[30] J. Palmer, Zeros of the Jimbo, Miwa, Ueno tau function, J. Math. Phys. 40 (1999), 6638-6681. MR:1725878 (2001g:34136)

[31] Y. Sibuya, Linear differential equations in the complex domain: Problems of analytic continuation, Transl. Math. Monogr., vol. 82, Amer. Math. Soc., Providence, RI, 1990. MR1084379 (92a:34010)

[32] V. S. Varadarajan, Linear meromorphic differential equations: A modern point of view, Bull. Amer. Math. Soc. 33 (1996), 1-42. MR1339809 (96h:34011)

[33] W. Wasow, Asymptotic expansions for ordinary differential equations, Robert E. Krieger Publishing Co., Huntington, NY, 1976. MR0460820 (57:812)

[34] X. Zhou, The Riemann-Hilbert problem and inverse scattering, SIAM J. Math. Anal. 20 (1989), 966-986. MR1000732 (90i:34046)

[35] A. A. Kapaev, Lax pairs for Painlevé equations, Isomonodromic Deformations and Applications in Physics (Montréal, QC, 2000), CRM Proc. Lecture Notes, vol. 31, Amer. Math. Soc., Providence, RI, 2002, pp. 37-48. MR1887346 (2003m:34216)

Steklov Mathematical Institute, Russian Academy of Sciences, Gubkina 8, Moscow 117966, GSP-1, RUSSIA

Department of Mathematical Sciences, Indiana University-Purdue University IndianapoLIS, INDIANAPOLIS, IN 46202-3216, USA

E-mail address: itsa@math.iupui.edu

St. Petersburg Branch, Steklov Mathematical Institute, Russian Academy of Sciences, Fontanka 27, St. Petersburg 191023, Russia

Received 6/OCT/2003

Originally published in English 